\title{
ON THE CONSTRUCTION OF MATRIX REPRESENTATIONS AND THEIR EXTENSIONS II
}

\author{
by ERIC B. KUISCH
}

(Received 8 April, 1990)

Introduction. In [5] we exhibited the construction of faithful irreducible matrix representations of $p$-groups $E$ and constructed their extensions to a semidirect product $E$. $H$, in case $E$ and $H$ satisfied suitable conditions. One of the major conditions was that the prime $p$ had to be odd.

In this paper we assume the same conditions as in [5], but now with $p=2$, in order to see if similar results can be obtained. Henceforth we will work with the following hypothesis.

Hypothesis. Let $G$ be a semidirect product of finite groups $E$ and $H$ where $E$ is a normal extraspecial 2-group of $G, H$ is a complement of $E$ in $G, C_{H}(E)=\{1\}$.

Under this hypothesis it is well known that $E$ has a unique (up to equivalence) faithful irreducible representation; say $D$.

In [1], Theorem 1.2, Dade showed that $D$ has an extension to $G$ if $H$ has a normal subgroup $K$ where $|K|$ is odd, and $[E, K]=E$. His proof is character theoretic and does not describe the matrix representation associated to the extension. It is therefore interesting to ask what the structure of this representation is.

As remarked in [5], the method developed to construct matrix representations and their extensions for the $p>2$-case cannot be applied in full generality to the $p=2$-case (see [5], for more details). Nevertheless we can build a projective extension of $D$ to $E$. Aut $(E)$, using the ideas of [5], and this projective representation is described in detail (see Theorem II.2.1).

We give a description of an extension in the $p=2$-case for a special situation which is suggested by Howlett's proof (see [2]) of Dade's theorem ([1, Theorem 1.2]). Howlett shows how the extension problem for the character case be reduced to the case where $H$ has a cyclic Sylow 2-subgroup. It is for this last situation that we will construct an extension of $D$ to $G$.

This paper is organized as follows.

$\S 0$ : We use the fact that there are only two types of extraspecial 2-groups (see [3, Satz III.13.8]); the structure of an extraspecial 2-group $E$ of the so-called first type is discussed and we construct a faithful irreducible representation of $E$. We note that it is well-known that all faithful irreducible representations of $E$ are equivalent.

$\S 1$ : A projective extension of the unique faithful irreducible representation of an extraspecial 2-group $E$ of first type to $E$. Aut $(E)$ is constructed.

§2: It is shown how for subgroups $H$ of $\operatorname{Aut}(E)$ ( $E$ of first type) with $|H|$ odd, the projective extension to $E$. $H$ (obtained by restriction) can be linearized (Theorem II.2.6).

§3: It is shown that Theorem II.2.6 can be generalized to hold for any extraspecial 2-group (Theorem II.3.13), by examining the extraspecial 2-groups of the second type.

§4: We construct an extension to $E . H$ where $H$ has cyclic Sylow 2-subgroup $C$ (Theorem II.4.3). This is done in two steps.

Step 1: an extension is constructed in case $H$ is a cyclic 2-group (Theorem II.4.1).

Glasgow Math. J. 33 (1991) 323-341. 
Step 2: we construct a 2-complement $K$ of $H$ ( $H$ is 2-nilpotent by [3, Satz IV.2.8]) and we build an extension to $E . H$ out of the extensions to $E . C$ and $E . K$ (Proposition (II.4.2)), using Theorem II.3.13.

Our notation is standard and is adapted from [3] and [4]. Additional notation will be introduced if necessary.

0. Preliminaries. We assume $E$ to be an extraspecial 2-group to $E / Z(E)$ is elementary abelian and $Z(E)$ is cyclic of order 2 . We investigate the semidirect product $G=E$. Aut $(E)$.

It is clear that $Z(E) \subseteq Z(G)$. By [3, Satz 13.8] there are two types of extraspecial 2-groups. The groups of the first type are central products of dihedral groups of order 8; each group of the second type is a central product of a quaternion group and a group of the first type.

In Sections $\S 0-\$ 2, E$ will be an extraspecial 2-group of first type.

An extraspecial 2-group $E$ of the first type has the following property.

There exist involutions $c, x_{i}, i=1, \ldots, 2 m$ such that every $x$ in $E$ can be uniquely written in the form $c^{a_{0}} x_{1}^{a_{1}} x_{2}^{a_{2}} \ldots x_{2 m}^{a_{2 m}}$ with $a_{i}=0$ or $1,0 \leq i \leq 2 m,\langle c\rangle=Z(E)$, and $|E / Z(E)|=2^{2 m}$, where the $x_{i}$ satisfy the following relations: $\left[x_{i}, x_{m+i}\right]=c$, if $1 \leq i \leq m$, and $\left[x_{j}, x_{k}\right]=1$ if $\{j, k\} \neq\{i, m+i\}$.

The elementary method of constructing irreducible faithful representations of $E$, as given in [5, Section 4] for odd primes $p$, can be performed in the present case as well. This leads to Theorem (II.0.1) which gives the unique (up to equivalence) non-linear faithful representation of $E$ in this way.

Notation. We will denote, for any $y \in E$, the coset $y Z(E)$ by $\bar{y}$.

The subgroup $E_{2}=\left\langle Z(E), x_{m+1}, \ldots, x_{2 m}\right\rangle$ is a maximal abelian normal subgroup of $E$ with index $2^{m}$. We have that $W:=\mathbb{C}\left[E_{2} / Z(E)\right]$ is a $2^{m}$-dimensional $\mathbb{C}$-vectorspace.

THEOREM II.0.1. Let $\phi$ be the bilinear map defined on $\mathbf{F}_{2}^{2 m} \times \mathbf{F}_{2}^{2 m}$ by $\phi(\alpha, \beta)=$ $\sum_{i=1}^{m} \alpha_{m+i} \beta_{i}$ with $\alpha=\left(\alpha_{1}, \ldots, \alpha_{2 m}\right), \beta=\left(\beta_{1}, \ldots, \beta_{2 m}\right)$. Define the map $D: E \rightarrow \operatorname{End}(W)$ by $D(x)(\bar{y})=(-1)^{a_{0}+\phi(x y, x)} \cdot\left(\overline{\left.x_{m+1}^{a_{m+1}} \cdot x_{m+2}^{a_{m+2}} \ldots x_{2 m}^{a_{2 m}} y\right)}\right.$; where $x=c^{a_{0}} x_{1}^{a_{1}} \cdot x_{2}^{a_{2}} \ldots x_{2 m}^{a_{2 m}}, \bar{y} \in W$. Then $D$ is an injective group homomorphism and it induces the unique (up to equivalence) faithful irreducible representation of $E$ which we also denote by $D$.

Proof. See [5, Theorem 4.6].

Definition. We denote by $A^{t}$ the transpose of the complex square matrix $A$.

Note that for any $x \in E$, the entries of $D(x)$ are \pm 1 . The following result can be easily derived from the definition of $D$.

COROLlaRY II.0.2. For all $x \in E$ we have that $D(x)^{t}=D\left(x^{-1}\right)=(D(x))^{-1}$.

1. Construction of a suitable transversal. We will show that a suitable transversal of $\operatorname{Inn}(E)$ in $\operatorname{Aut}(E)$ can be constructed which gives us the possibility to exhibit a projective extension of the faithful representation $D$ of $E$, in the spirit of [5]. For convenience we introduce some notation. 
Notation. (1) For $a \in \mathbb{Z}$ define $\bar{a}=a \bmod 2$. Let $\alpha \in \mathbf{F}_{2}^{2 m}, \alpha=\left(\bar{a}_{1}, \bar{a}_{2}, \ldots, \overline{a_{2 m}}\right)$, $a_{i} \in \mathbb{Z}$. Then $x^{\alpha}$ is defined as $x_{1}^{a_{1}} x_{2}^{a_{2}} \ldots x_{2 m}^{a_{2 m}}$.

(2) Let $\gamma \in \mathbf{F}_{2}^{2 m}$. Then $\mathbf{i}(\gamma)$ is the inner automorphism of $E$ determined by $\mathbf{i}(\gamma)(y)=x^{\gamma} y\left(x^{\gamma}\right)^{-1}$ for all $y \in E$.

(3) Define the bilinear map $\phi$ on $\mathbf{F}_{2}^{2 m} \times \mathbf{F}_{2}^{2 m}$ by $\phi(\alpha, \beta)=\sum_{i=1}^{m} \alpha_{m+i} \beta_{i}$, with $\alpha=$ $\left(\alpha_{1}, \ldots, \alpha_{2 m}\right), \beta=\left(\beta_{1}, \ldots, \beta_{2 m}\right)$. By abuse of notation we define, for $z, z^{\prime} \in Z(E)$, $\phi\left(z x^{\alpha}, z^{\prime} x^{\beta}\right)$ to be $\phi(\alpha, \beta)$.

(4) Let $T \in G L(2 m, 2)$. Then $C_{T}=\left|\left\{\alpha \in \mathbb{F}_{2}^{2 m} \mid T \alpha=\alpha\right\}\right|$.

Quadratic forms over $\mathbf{F}_{2}$ will play an important role in our investigations; therefore we recall their definition.

Definition II.1.0. Let $V$ be a vector space defined over a field $K$. Then a quadratic form on $V$ is defined as a map $\tau: V \rightarrow K$ satisfying the following conditions

(i) $\tau(c v)=c^{2} \tau(v)$, for all $c \in K, v \in V$;

(ii) the map $B_{\tau}: V \times V \rightarrow K$, defined by

$$
B_{\tau}(v, w)=\tau(v+w)-\tau(v)-\tau(w)
$$

is a symmetric bilinear form.

See [6, p. 24] for more details.

Finally we define a special quadratic form $q$ on $\mathbf{F}_{2}^{2 m}$ by

$$
q(\alpha)=\phi(\alpha, \alpha)=\sum_{i=1}^{m} \alpha_{m+i} \alpha_{i}, \text { where } \alpha=\left(\alpha_{1}, \alpha_{2}, \ldots, \alpha_{2 m}\right)
$$

We denote the symmetric bilinear form $B_{q}$ by $f$.

Lemma II.1.1. Let $\theta \in \operatorname{Aut}(E)$. Then for each $\alpha, \theta\left(x^{\alpha}\right)=t$. $x^{\alpha^{\prime}}$ for unique elements $t \in Z(E)$ and $\alpha^{\prime} \in \mathbf{F}_{2}^{2 m}$. The map $T: \mathbf{F}_{2}^{2 m} \rightarrow \mathbf{F}_{2}^{2 m}$ given by $T(\alpha)=\alpha^{\prime}$, is a linear transformation preserving the quadratic form $q$.

Proof. The map $T$ is bijective and linear, as $\theta \in \operatorname{Aut}(E)$. Moreover $T$ fixes $q$, as shown by the following argument. As $E / Z(E)$ is elementary abelian, $\left(x^{\alpha}\right)^{2} \in Z(E)$ and therefore $\left(x^{\alpha}\right)^{2}=\theta\left(\left(x^{\alpha}\right)^{2}\right)=\left(\theta\left(x^{\alpha}\right)\right)^{2}$. Also $\left(x^{\alpha}\right)^{2}=c^{\phi(\alpha, \alpha)}$ and $\left(\theta\left(x^{\alpha}\right)\right)^{2}=\left(x^{T \alpha}\right)^{2}=$ $c^{\phi(T \alpha, T \alpha)}$ [see I.4.6.1]. So $q(\alpha)=\phi(\alpha, \alpha)=\phi(T \alpha, T \alpha)=q(T(\alpha))$. This proves the lemma.

Every element of $\operatorname{Aut}(E)$ induces a linear transformation on the $2 m$-dimensional $\mathbf{F}_{2}$-vectorspace $E / Z(E)$. We just saw that this linear transformation is an element of the group consisting of all those linear transformations on a $2 m$-dimensional $\mathbf{F}_{2}$-vectorspace, that preserve the quadratic form $q$. We denote this group by $O_{1}(2 m, 2)$. We now show how for any element $T$ of $O_{1}(2 m, 2)$ an automorphism of $E$ can be constructed that fixes $Z(E)$ and induces $T$ as a linear transformation on the $\mathbf{F}_{2}$-vectorspace $E / Z(E)$. Hence $\operatorname{Aut}(E) / \operatorname{Inn}(E) \cong O_{1}(2 m, 2)$.

THEOREM II.1.2. Let $T \in O_{1}(2 m, 2)$. Define the map $\mu_{T}^{\prime}: E \rightarrow E$ by

$$
\mu_{T}^{\prime}\left(c^{a_{0}} x_{1}^{a_{1}} x_{2}^{a_{2}} \ldots x_{2 m}^{a_{2 m}}\right)=c^{a_{0}} c^{q_{T}(\alpha)} x_{1}^{a_{1}^{i}} x_{2}^{a_{2}^{i}} \ldots x_{2 m}^{a_{2 m}}
$$

where $T(\alpha)=T\left(a_{1}, a_{2}, \ldots, a_{2 m}\right)=\left(a_{1}^{\prime}, a_{2}^{\prime}, \ldots, a_{2 m}^{\prime}\right)$, and $q_{T}(\alpha)=\sum_{i<j} \phi\left(k_{i}, k_{j}\right) a_{i} a_{j}$, with $k_{i}=T\left(e_{i}\right)$ where $\left\{e_{i}=(\underbrace{0, \ldots, 0}_{i-1}, 1,0, \ldots, 0) \mid 1 \leq i \leq 2 m\right\}$ is the standard basis of $\mathbf{F}_{2}^{2 m}$. 
Then $\mu_{T}^{\prime}$ is an element of $\operatorname{Aut}(E)$ and it induces $T$ as a linear transformation on $E / Z(E)$.

Proof. We need to show that

$$
\mu_{T}^{\prime}\left(x^{\alpha}\right) \cdot \mu_{T}^{\prime}\left(x^{\beta}\right)=\mu_{T}^{\prime}\left(x^{\alpha} \cdot x^{\beta}\right)
$$

Note that $\mu_{T}^{\prime}\left(x^{\alpha} x^{\beta}\right)=c^{\phi(\alpha, \beta)} \cdot \mu_{T}^{\prime}\left(x^{\alpha+\beta}\right)=c^{\phi(\alpha, \beta)} c^{q T(\alpha+\beta)} x^{T(\alpha+\beta)}$.

The proof can now be derived in the following way. By definition of $\mu_{T}^{\prime}$, $\mu_{T}^{\prime}\left(x^{\alpha}\right) \cdot \mu_{T}^{\prime}\left(x^{\beta}\right)=c^{q_{T}(\alpha)+q_{T}(\beta)+\phi(T(\alpha), T(\beta))} \cdot x^{T(\alpha+\beta)}$; furthermore

$$
\begin{aligned}
\phi(T(\alpha), T(\beta))= & \phi\left(\sum a_{i} T\left(e_{i}\right), \sum b_{i} T\left(e_{i}\right)\right) \\
= & \sum_{i, j} \phi\left(T\left(e_{i}\right), T\left(e_{j}\right)\right) a_{i} b_{j} \\
= & \sum_{i<j}\left(\phi\left(T\left(e_{i}\right), T\left(e_{j}\right)\right) a_{i} b_{j}+\phi\left(T\left(e_{j}\right), T\left(e_{i}\right)\right) a_{j} b_{i}\right) \\
& {\left[\operatorname{as} \phi\left(T\left(e_{i}\right), T\left(e_{i}\right)\right)=\phi\left(e_{i}, e_{i}\right)=0 \text { as } T \in O_{1}(2 m, 2)\right] } \\
= & \sum_{i<j} \phi\left(T\left(e_{i}\right), T\left(e_{j}\right)\right)\left(a_{i} b_{j}+a_{j} b_{i}\right)+\phi(\alpha, \beta) \\
& {\left[\operatorname{as} \phi\left(T\left(e_{i}\right), T\left(e_{j}\right)\right)+\phi\left(T\left(e_{j}\right), T\left(e_{i}\right)\right)=f\left(T\left(e_{j}\right), T\left(e_{i}\right)\right)=f\left(e_{j}, e_{i}\right)=0\right.} \\
& \text { unless } j-i=m] .
\end{aligned}
$$

So $q_{T}(\alpha)+q_{T}(\beta)+\phi(T(\alpha), T(\beta))=q_{T}(\alpha+\beta)+\phi(\alpha, \beta)$. This proves that $\mu_{T}^{\prime}$ is a homomorphism; as $T$ is bijective we find that $\mu_{T}^{\prime} \in \operatorname{Aut}(E)$. By definition $\mu_{T}^{\prime}$ induces $T$. This proves the theorem.

The proof of [5, Theorem 5.13) can be used to obtain the following result which will be denoted in the sequel by (TH).

(TH) Let $G$ be a finite group, $N$ a non-abelian normal subgroup of $G$. Put $A=N / Z(N)$ and let $\left\{x_{a} \mid a \in A\right\}$ be a transversal for $Z(N)$ in $N$. Assume that

(i) $Z(N) \subseteq Z(G)$.

(ii) $\Xi$ is a $G$-invariant irreducible representation of $N$, defined over a splitting field $k$ of $k[N]$ with $\operatorname{char}(k) \nmid|N|$,

(iii) $\operatorname{tr}(\Xi)=0$ on $N-Z(N)$.

Let $\mathfrak{I}=\left\{g_{i}|i=1, \ldots| G:, N \mid\right\}, g_{1}=1$, be a transversal for $N$ in $G$. Define the map $\mathfrak{N}_{\mathfrak{T}}: G \rightarrow G L(\operatorname{deg}(\Theta), k)$ by

$$
\mathfrak{R}_{\mathfrak{T}}\left(n g_{i}\right)=\Xi(n)\left(\frac{1}{\left|C_{A}\left(g_{i}\right)\right|} \sum_{a \in A} \Xi\left(g_{i} x_{a} g_{i}^{-1} x_{a}^{-1}\right)\right)
$$

where $g_{i} \in \mathfrak{I}$, and $n \in N$. Then the following holds.

(a) If $\mathfrak{R}$ is some transversal for $N$ in $G$ with $1 \in \mathfrak{A}$ and $C_{A}(t)=C_{N}(t) / Z(N)$ for all $t \in \Re$, then $\Re_{\Re}$, defined as in $(*)$, is a projective $k$-representation of $G$ extending $\Xi$.

(b) Suppose that $N / Z(N)$ is abelian and $\Xi$ is faithful. Then out of the transversal $\mathfrak{I}$ and the group structure of $N / Z(N)$, a transversal $\mathfrak{P}$ can be determined for which $\mathfrak{R}_{\mathfrak{P}}$ is a projective representation of $G$, extending $\Xi$. Moreover, for every $g_{i} \in \mathfrak{B}$ we have that $C_{A}\left(g_{i}\right)=C_{N}\left(g_{i}\right) / Z(N)$. 
REMARK. In the sequel we will apply part (b) of this theorem with $k=\mathbb{C}$ and $N$ an extraspecial 2-group. Observe that if (TH)(i)-(iii) holds and $(|N|,|G / N|)=1$, then there indeed exists a transversal $\mathfrak{R}$ for $N$ in $G$ with $1 \in \mathfrak{R}$ and $C_{A}(t)=C_{N}(t) / Z(N)$ for all $t \in \mathfrak{R}$. This follows from Theorem 6.2.2 in Gorenstein's book Finite Groups (1980). So in this situation we can conclude from (a) that $\mathfrak{N}_{\Re}$, defined as in $(*)$, is a projective $k$-representation of $G$ extending $\Xi$, and then a result of $H$. E. Becker [Archiv der Mathematik, 27 (1976), 588-592, Lemma 1] can be applied to linearize $\mathfrak{N}_{\Re}$ in order to calculate an explicit extension of $\Xi$. The following lemma will enable us to apply (TH), as it shows that $\mu_{T}^{\prime}$ can be modified in such a way that the condition of (TH(a)) is satisfied. Recall that by Notation (2) any $\beta \in \mathbf{F}_{2}^{2 m}$, affords an inner automorphism $\mathbf{i}(\beta)$ of $E$.

Lemma II.1.3. Let $T \in O_{1}(2 m, 2)$ and let $\mu_{T}^{\prime}$ be defined as in Theorem II.1.2. Then there exists some $\rho_{T} \in \mathbf{F}_{2}^{2 m}$ such that

(a) $\mathbf{i}\left(\rho_{T}\right) \mu_{T}^{\prime}$ is an automorphism of $E$ which induces $T$, and

(b) $\mathrm{i}\left(\rho_{T}\right) \mu_{T}^{\prime}(x)=t x$ for some $x$ with $t \in Z(E)$ only if $t=1$.

Proof. Put $F=\left\{x \in E \mid \mu_{T}^{\prime}(x)=t x, t \in Z(E)\right\}$. Then for $x \in F$, we have $x=c^{a} x^{\alpha}$ and $T(\alpha)=\alpha$. There are two possibilities. The first is that $q_{T}(\alpha)=0$ for all $\alpha$ with $T(\alpha)=\alpha$. In this case $\mathrm{i}\left(\rho_{T}\right)$ can be chosen to be the identity automorphism of $E$. The second possibility is that there exists some $x^{\alpha} \in F$ with $q_{T}(\alpha)=1$. Put $F_{0}:=\left\{x \in E \mid \mu_{T}^{\prime}(x)=x\right\}$. Then $F=F_{0} \cup x^{\alpha} F_{0}$. Let $F_{0} / Z(E)=\left\langle x^{\beta_{i}} Z(E): 1 \leq i \leq n\right\rangle$ and $F / Z(E)=$ $\left\langle x^{\alpha} Z(E), F_{0} / Z(E)\right\rangle$, for some integer $n$. Any inner automorphism of $\gamma$ of $E$, fixes every coset $y Z(E), y \in E$, as $E^{\prime}=Z(E)$. Hence if $\left\{x^{\alpha_{1}} Z\left(E, \ldots, x^{\alpha_{2 m}} Z(E)\right\}\right.$ is a basis for $E / Z(E)$, we get that $\gamma\left(x^{\alpha_{i}}\right)=c_{i} x^{\alpha_{i}}$, with $c_{i} \in Z(E), 1 \leq i \leq 2 m$. As $|\operatorname{Inn}(E)|=2^{2 m}$, and $|Z(E)|=2$, we have that for any choice of central elements $d_{i}, i=1, \ldots, 2 m$ there exists an inner automorphism $\gamma^{\prime}$ such that

$$
\gamma^{\prime}\left(x^{\alpha_{i}}\right)=d_{i} x^{\alpha_{i}}, \quad i=1, \ldots, 2 m \text {. }
$$

In particular we have that some inner automorphism $\mathbf{i}\left(\rho_{T}\right)$ exists such that

$$
\mathbf{i}\left(\rho_{T}\right)\left(x^{\alpha}\right)=c \cdot x^{\alpha}, \quad \mathbf{i}\left(\rho_{T}\right)\left(x^{\beta_{i}}\right)=x^{\beta_{i}}, \quad 1 \leq i \leq n .
$$

We have that $\mathbf{i}\left(\rho_{T}\right) \mu_{T}^{\prime}$ induces $T$ on $E / Z(E)$. If $\mathbf{i}\left(\rho_{T}\right) \mu_{T}^{\prime}(x)=t x$ for some $x$ with $t \in Z(E)$ then $\mu_{T}^{\prime}(x)=s x$, for some $s \in Z(E)$, hence $x \in F$. If $x \in F_{0}$, then $x$ is fixed by $i\left(\rho_{T}\right) \mu_{T}^{\prime}$; otherwise $x=x^{\alpha} . y$ with $y \in F_{0}$ and $\mathbf{i}\left(\rho_{T}\right) \mu_{T}^{\prime}(x)=\mathbf{i}\left(\rho_{T}\right)\left(\mu_{T}^{\prime}\left(x^{\alpha}\right) \cdot y\right)=c \cdot x^{\rho_{T}} x^{\alpha}\left(x^{\rho_{T}}\right)^{-1} y=$ $x^{\alpha} y=x$. This proves the lemma.

From now on we assume that elements $\mathrm{i}\left(\rho_{T}\right)$ have been chosen such as to satisfy the lemma above, and denote $\mathrm{i}\left(\rho_{T}\right) \mu_{T}^{\prime}$ by $\mu_{T}$. Note that $\mu_{T}\left(x^{\alpha}\right)=c^{q_{T}(\alpha)+f\left(\rho_{T}, T \alpha\right)} x^{T(\alpha)}$.

An application of (TH) immediately gives that the matrix $P\left(\mu_{T}\right)$ defined by

$$
P\left(\mu_{T}\right)=\frac{1}{c_{T}} \sum_{\alpha \in \mathbf{F}_{2}^{2 m}}(-1)^{q_{T}(\alpha)+f\left(\rho_{r}, T(\alpha)\right)+\phi(T \alpha+\alpha, \alpha)} \cdot D\left(x^{(T+1)(\alpha)}\right)
$$

is invertible and satisfies $P\left(\mu_{T}\right)^{-1} D(x) P\left(\mu_{T}\right)=D\left(\mu_{T}^{-1}(x)\right)$.

We now study the relation between $P\left(\mu_{T}\right)$ and $P\left(\mu_{T^{-1}}\right)$. The following holds There is some $\beta \in \mathbf{F}_{2}^{2 m}$ such that

$$
x^{\alpha}\left(\mu_{T}\left(x^{\alpha}\right)\right)^{-1}=c^{f(\beta, \alpha)} \cdot \mu_{T^{-1}}\left(x^{T \alpha}\right)\left(x^{T \alpha}\right)^{-1} \text { for all } \alpha \in \mathbf{F}_{2}^{2 m} .
$$


Indeed, working out the left-hand side of the equation gives

whereas

$$
x^{\alpha}\left(\mu_{T}\left(x^{\alpha}\right)\right)^{-1}=c^{q_{T}(\alpha)+f\left(\rho_{T}, T \alpha\right)+\phi(\alpha+T \alpha, T \alpha)} \cdot x^{\alpha+T \alpha}
$$

$$
\mu_{T^{-1}}\left(x^{T \alpha}\right)\left(x^{T \alpha}\right)^{-1}=c^{q_{T^{-1}}(T \alpha)+f\left(\rho_{\left.T^{-1}, \alpha\right)+\phi(\alpha+T \alpha, T \alpha)}\right.} \cdot x^{\alpha+T \alpha} .
$$

By II.1.2.1 we have for all $T \in O_{1}(2 m, 2)$ that $\phi(T(\alpha), T(v))=B_{T}(\alpha, v)+\phi(\alpha, v)$ where $B_{T}$ is the bilinear form satisfying $B_{T}(\alpha, v)=q_{T}(\alpha)+q_{T}(v)+q_{T}(\alpha+v)$. Now

$$
\begin{aligned}
\phi(\alpha, v) & =\phi\left(T^{-1}(T \alpha), T^{-1}(T v)\right)=B_{T^{-1}}(T \alpha, T v)+\phi(T \alpha, T v) \\
& =B_{T^{-1}}(T \alpha, T v)+B_{T}(\alpha, v)+\phi(\alpha, v) .
\end{aligned}
$$

Conclusion: $B_{T^{-1}}(T \alpha, T v)=B_{T}(\alpha, v)$.

As we have that $q_{T}(\alpha)+q_{T}(v)=B_{T}(\alpha, v)+q_{T}(\alpha+v)$ we see that the form $q_{T}(\alpha)+q_{T^{-1}}(T \alpha)$ is linear. This gives the result.

The following lemma is a consequence of (II.1.3.1).

Lemma (II.1.4). Let $T \in O_{1}(2 m, 2)$ then

$$
P\left(\mu_{T}\right)^{t}=A^{-1} P\left(\mu_{T^{-1}}\right) A \text { with } A=D\left(x^{\sigma}\right) \text { for some suitable } \sigma \in \mathbf{F}_{2}^{2 m} .
$$

Proof. By definition of $P\left(\mu_{T}\right)$ and using II.0.2 we have that

$$
\begin{aligned}
& P\left(\mu_{T}\right)^{t}=\frac{1}{c_{T}} \sum_{\alpha \in \mathbf{F}_{2}^{2 m}} D\left(\mu_{T} \cdot x^{\alpha} \cdot \mu_{T}^{-1} \cdot\left(x^{\alpha}\right)^{-1}\right)^{t} \\
& =\frac{1}{c_{T}} \sum_{\alpha \in \mathbf{F}_{2}^{2 m}} D\left(\mu_{T} \cdot x^{\alpha} \cdot \mu_{T}^{-1} \cdot\left(x^{\alpha}\right)^{-1}\right)^{-1} \\
& =\frac{1}{c_{T}} \sum_{\alpha \in \mathbf{F}_{2}^{2 m}}(-1)^{f(\beta, \alpha)} D\left(\mu_{T^{-1}} \cdot x^{T \alpha} \cdot\left(\mu_{T^{-1}}\right)^{-1} \cdot\left(x^{T \alpha}\right)^{-1}\right) \quad \text { (by (II.1.3.1)) }
\end{aligned}
$$

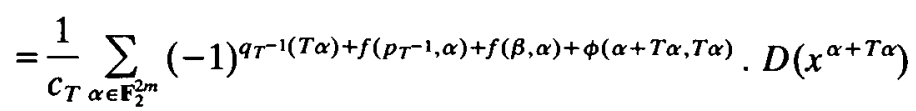

By definition of $P\left(\mu_{T}\right)$ the quadratic form $q^{\prime}(\alpha)=q_{T^{-1}}(T \alpha)+f\left(\rho_{T^{-1}}, \alpha\right)+$ $\phi(\alpha+T \alpha, T \alpha)$ has the following property:

$$
q^{\prime}(\alpha+\zeta)=q^{\prime}(\alpha) \text { if } \zeta=T(\zeta)
$$

For such $\zeta$ we have that $f(\beta, \alpha+\zeta)=f(\beta, \alpha)$. This follows as $1=x^{\zeta}\left(\mu_{T}\left(x^{\zeta}\right)^{-1}=\right.$ $c^{f(\beta, \zeta)} \cdot\left(\mu_{T^{-1}}\left(x^{T \zeta}\right)\right)\left(x^{T \zeta}\right)^{-1}=c^{f(\beta, \zeta)}$, using II.1.3(b).

By an observation similar to [5, proof of Lemma (6.2)]), we conclude that there exists a linear mapping $L: \operatorname{Im}(T+I) \rightarrow \mathbf{F}_{2}^{2 m}$ such that

where

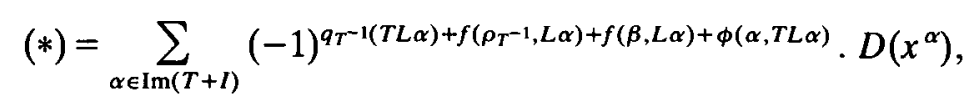

$$
\sum_{\alpha \in \operatorname{Im}(T+l)}(-1)^{q_{T^{-1}}(T L \alpha)+f\left(\rho_{\left.T^{-1}, L \alpha\right)+\phi(\alpha, T L \alpha)}\right.} \cdot D\left(x^{\alpha}\right)=P\left(\mu_{T^{-1}}\right)
$$


Now there is some $\sigma$ such that $f(\beta, L \alpha)=f(\sigma, \alpha)$ for all $\alpha$, as the map $\alpha \rightarrow f(\beta, L(\alpha))$ is a functional. For this choice of $\sigma$, we get that $A:=D\left(x^{\sigma}\right)$ satisfies the statement of the lemma.

This finishes the proof of the lemma.

Let $T, S \in O_{1}(2 m, 2)$. As any automorphism of $E$ which fixes every coset $x Z(E)$ is inner, we have that $\mu_{T} \mu_{S}=h . \mu_{T S}$ where $h \in \operatorname{Inn}(E)$. Choose $\beta \in \mathbf{F}_{2}^{2 m}$, such that $h=\mathbf{i}(\beta)$. Using the definition of the matrices $P\left(\mu_{T}\right), T \in O_{1}(2 m, 2)$, we conclude that

$$
\begin{aligned}
\left(P\left(\mu_{T}\right) P\left(\mu_{S}\right)\right) . D(x) & \cdot\left(P\left(\mu_{T}\right) P\left(\mu_{S}\right)\right)^{-1}=D\left(\mu_{T} \mu_{S}(x)\right) \\
= & D\left(x^{\beta}\right) D\left(\mu_{T S}(x)\right) D\left(x^{\beta}\right)^{-1}=\left(D\left(x^{\beta}\right) P\left(\mu_{T S}\right)\right) . D(x) \cdot\left(D\left(x^{\beta}\right) P\left(\mu_{T S}\right)\right)^{-1} .
\end{aligned}
$$

As $D$ is an irreducible representation of $E$, Schur's lemma applies, and we get that

$$
P\left(\mu_{T}\right) P\left(\mu_{S}\right)=\lambda . D\left(x^{\beta}\right) P\left(\mu_{T S}\right) \text { for some } \lambda \in \mathbb{C}^{*} \text {. }
$$

The following theorem shows what the value of $\lambda$ is when $S=T^{-1}$. As in [5], we define $\mathrm{CO}_{T}=$ codimension of the eigenspace of $T$ associated with the eigenvalue 1 , for $T \in G L(2 m, 2)$.

Theorem (II.1.5). Let $T \in O_{1}(2 m, 2)$. Then

$\operatorname{det}\left(P\left(\mu_{T}\right)\right)= \pm 2^{2^{m-1} \cdot \text { cor }_{T}}$ and $P\left(\mu_{T}\right) P\left(\mu_{T^{-1}}\right)= \pm 2^{\text {corT }_{T}} . D\left(x^{\gamma}\right)$ for some $\gamma \in \mathbf{F}_{2}^{2 m}$.

Proof. We compute the product $P\left(\mu_{T}\right) P\left(\mu_{T^{-1}}\right)$. By definition of $P\left(\mu_{T}\right)$ we find that

where

$$
P\left(\mu_{T}\right) P\left(\mu_{T^{-1}}\right)=\left(1 / c_{T}\right)^{2} \sum_{\alpha, \beta}(-1)^{k(\alpha, \beta)} \cdot D\left(x^{(T+l)(\alpha)}\right) . D\left(x^{\left(T^{-1}+l\right)(\beta)}\right),
$$

$$
\begin{gathered}
k(\alpha, \beta)=q_{T}(\alpha)+f\left(\rho_{T}, T \alpha\right)+\phi((T+I)(\alpha), \alpha)+q_{T^{-1}}(\beta) \\
+f\left(\rho_{T^{-1}}, T^{-1} \beta\right)+\phi\left(\left(T^{-1}+I\right)(\beta), \beta\right) .
\end{gathered}
$$

As $P\left(\mu_{T}\right) P\left(\mu_{T^{-1}}\right)=\lambda . D\left(x^{\gamma}\right)$, for some $\gamma \in \mathbf{F}_{2}^{2 m}$, we find by equating the coefficients of $D\left(x^{\gamma}\right)$ on both sides of the equation (using that the set $\left\{D\left(x^{\alpha}\right) \mid \alpha \in \mathbf{F}_{2}^{2 m}\right\}$ is linearly independent) that for every $\alpha$, we only need to consider those $\beta$ for which $\left(T^{-1}+I\right)(\beta)=$ $(T+I)(\alpha)+\gamma$.

So then $\gamma \in \operatorname{Im}(T+I)$, say $\gamma=T+I(v)$, and for any such pair $\{\alpha, \beta\}$ we find that $\beta=T(\alpha+v)+\zeta$ with $T(\zeta)=\zeta$. We recall the following facts

I) $q_{T^{-1}}(\zeta)=f\left(\rho_{T^{-1}}, \zeta\right)$, and $q_{T}(\alpha)+q_{T^{-1}}(T(\alpha))=f(\tau, \alpha)$ for some $\tau \in \mathbf{F}_{2}^{2 m}$, Now

II) $\phi(\alpha, \zeta)=B_{T^{-1}}(T \alpha, \zeta)+\phi(T \alpha, \zeta)$ if $T \zeta=\xi$ (by formula (II.1.3.2)).

$$
\begin{aligned}
k(\alpha, \beta)= & q_{T}(\alpha)+f\left(\rho_{T}, T \alpha\right)+\phi(T+I(\alpha), \alpha)+q_{T^{-1}}(T(\alpha+v)+\zeta)+f\left(\rho_{T^{-1}}, \alpha+v+\zeta\right) \\
& +\phi(T(\alpha)+\alpha+\gamma, T(\alpha+v)+\zeta) \\
= & q_{T}(\alpha)+f\left(\rho_{T}, T \alpha\right)+\phi(T+I(\alpha), T+I(\alpha))+q_{T^{-1}}(T(\alpha))+q_{T^{-1}}(\zeta) \\
& +B_{T^{-1}}(T \alpha, \zeta)+B_{T^{-1}}(T v, \zeta)+B_{T^{-1}}(T \alpha, T v)+q_{T^{-1}}(T(v))+f\left(\rho_{T^{-1}}, \alpha+\zeta\right) \\
& +f\left(\rho_{T^{-1}}, v\right)+\phi(T(\alpha)+\alpha, \zeta)+\phi(T(\alpha)+\alpha, T v)+\phi(\gamma, T(\alpha+v)+\zeta) \\
\text { by I) } & f(\tau, \alpha)+f\left(\rho_{T}, T \alpha\right)+f\left(\rho_{T^{-1}}, \alpha\right)+\phi(T+I(\alpha), T+I(\alpha))+B_{T^{-1}}(T \alpha, \zeta) \\
& +B_{T^{-1}}(T v, \zeta)+B_{T^{-1}}(T \alpha, T v)+q_{T^{-1}}(T(v))+f\left(\rho_{T^{-1}}, v\right)+\phi(T(\alpha)+\alpha, \zeta) \\
& +\phi(T(\alpha)+\alpha, T v)+\phi(\gamma, T(\alpha+v)+\zeta)
\end{aligned}
$$




$$
\begin{aligned}
& \stackrel{\text { by II) }}{=} f(\tau, \alpha)+f\left(\rho_{T}, T \alpha\right)+f\left(\rho_{T^{-1}}, \alpha\right)+\phi(T+I(\alpha), T+I(\alpha))+B_{T^{-1}}(T \alpha, T v) \\
& \quad+q_{T^{-1}}(T(v))+f\left(\rho_{T^{-1}}, v\right)+\phi(T(\alpha)+\alpha, T v)+\phi(\gamma, T(\alpha+v)) \\
& =L(\alpha)+\phi(T+I(\alpha), T+I(\alpha))+d_{T}
\end{aligned}
$$

where $L$ is a functional and $d_{T}=f\left(\rho_{T^{-1}}, v\right)+\phi(\gamma, T v)+q_{T^{-1}}(T(v))$. So therefore

$$
\left(\mu_{T^{-1}}\left(x^{T v}\right)\right)\left(x^{T v}\right)^{-1}=(-1)^{d_{T}} \cdot x^{\gamma}
$$

and we conclude that

$$
\left(1 / c_{T}\right)^{2} \cdot \sum_{\alpha} \sum_{\zeta \in \operatorname{Fix}(T)}(-1)^{k(\alpha, T(\alpha+v)+\zeta)} \cdot D\left(x^{(T+I)(\alpha)}\right) \cdot D\left(x^{\left(T^{-1}+I\right)(T(\alpha+v)+\zeta)}\right)=\lambda \cdot D\left(x^{\gamma}\right)
$$

Therefore

$$
\begin{aligned}
\lambda & =\left(1 / c_{T}\right)^{2} \cdot \sum_{\alpha} \sum_{\zeta \in \mathrm{Fix}(T)}(-1)^{\phi(\alpha+T \alpha, \gamma)+L(\alpha)+d_{T}} \\
& =\left(1 / c_{T}\right)(-1)^{d_{T}} \sum_{\alpha}(-1)^{\phi(\alpha+T \alpha, \gamma)+L(\alpha)} .
\end{aligned}
$$

The exponent of -1 is a functional so $\sum_{\alpha}(-1)^{\phi(\alpha+T \alpha, \gamma)+L(\alpha)}=0$ or $2^{2 m}$. The first possibility doesn't hold as $\lambda \neq 0$. Apparently

$$
\lambda=\left(1 / c_{T}\right)(-1)^{d_{T}} \cdot 2^{2 m}=(-1)^{d^{2}} 2^{\mathrm{co}_{T}} .
$$

Now it follows immediately that

$$
\operatorname{det}\left(P\left(\mu_{T}\right) P\left(\mu_{T^{-1}}\right)\right)=\operatorname{det}\left(P\left(\mu_{T}\right)\right)^{2} \quad(\text { by II.1.4 })=2^{\text {co } \cdot 2^{m}} \cdot \operatorname{det}\left(D\left(x^{\gamma}\right)\right) .
$$

As $\operatorname{det}\left(P\left(\mu_{T}\right)\right)$ is integral, for $P\left(\mu_{T}\right)$ is a sum of matrices with integral coefficients, and as $\operatorname{det}\left(D\left(x^{\gamma}\right)\right)= \pm 1$, we have $\operatorname{det}\left(P\left(\mu_{r}\right)\right)= \pm 2^{2^{m-1} \cdot \cos _{T}}$ (and $\operatorname{det}\left(D\left(x^{\gamma}\right)\right)=1$ ). This proves the theorem.

In order to proceed with our investigation we now need a result on quadratic forms.

LEMMA II.1.6. Let $r$ be a quadratic form on a $\mathbf{F}_{2}$-vectorspace $V$ then $\sum_{v \in V}(-1)^{r(v)}=0$ or $\pm 2^{k}$ for some positive integer $k$.

Proof. As $r$ is a quadratic form we have that the map $Q: V \times V \rightarrow \mathbf{F}_{2}$ defined by $Q(v, w)=r(v)+r(w)+r(v+w)$, is a bilinear form. Now

$$
\begin{aligned}
\left(\sum_{v \in V}(-1)^{r(v)}\right)^{2} & =\sum_{v \in V}(-1)^{r(v)} \cdot \sum_{w \in V}(-1)^{r(v+w)} \\
& =\sum_{w \in V} \sum_{v \in V}(-1)^{r(w)}(-1)^{Q(v, w)} .
\end{aligned}
$$

As $Q(, w)$ is linear $\sum_{v \in V}(-1)^{Q(v, w)}=2^{\operatorname{dim}(V)}$ if $Q(, w)$ is identically zero on $V$, and $\sum_{v \in V}(-1)^{Q(v, w)}=0$ otherwise. We introduce the set $W=\{w \in V \mid Q(, w)=0\}$. 
Observe that $W$ is a subspace of $V$, and that $r$ is linear on $W$. Hence

$$
\left(\sum_{v \in V}(-1)^{r(v)}\right)^{2}=\sum_{w \in W}(-1)^{r(w)} \cdot 2^{\operatorname{dim}(V)}
$$

and we conclude that $\left(\sum_{v \in V}(-1)^{r(v)}\right)^{2}=0$ or $2^{\operatorname{dim}(w)+\operatorname{dim}(V)}$.

As $\sum_{v \in V}(-1)^{r(v)}$ is integral we find that $\sum_{v \in V}(-1)^{r(v)}=0$, or $\operatorname{dim}(W)+\operatorname{dim}(V)=2 e$ for some integer $e$ and $\sum_{v \in V}(-1)^{r(v)}= \pm 2^{e}$. This proves the lemma.

Lemma II.1.6 has the following important consequence.

Theorem II.1.7. Let $T, S \in O_{1}(2 m, 2)$. Then $P\left(\mu_{T}\right) P\left(\mu_{S}\right)= \pm 2^{e} . D\left(x^{\beta}\right) P\left(\mu_{T S}\right)$ for some positive integer $e$, where $\beta$ is such that $\mu_{T} \mu_{S}=\mathrm{i}(\beta) . \mu_{T S}$.

Proof. The set $\left\{D\left(x^{\alpha}\right) \mid \alpha \in \mathbf{F}_{2}^{2 m}\right\}$ is linearly independent; so equating the coefficients of $D\left(x^{\beta}\right)$ on both sides of the equation $P\left(\mu_{T}\right) P\left(\mu_{S}\right)=\lambda . D\left(x^{\beta}\right) P\left(\mu_{T S}\right)$, using (II.1.4.1), yields that $\lambda=\sum_{\alpha \in \mathcal{V}+\delta}(-1)^{r(\alpha)}$ where

$$
\begin{aligned}
r(\alpha)=q_{r}\left(T^{-1} N \alpha\right)+f\left(\rho_{T}, N \alpha\right)+ & \phi\left(\alpha, T^{-1} N \alpha\right)+q_{s}\left(S^{-1} M \alpha+\beta\right) \\
& +f\left(\rho_{S}, M \alpha+\beta\right)+\phi\left(\alpha+\beta, s^{-1} M \alpha+\beta\right)+\phi(\alpha, \alpha+\beta),
\end{aligned}
$$

$N$ and $M$ are linear mappings (see II.1.4.1), $\mathscr{V}:=\operatorname{Im}(T+I) \cap \operatorname{Im}(S+I)$ ) and $\mathscr{V}+\delta=$ $\operatorname{Im}(T+I) \cap(\operatorname{Im}(S+I)+\beta)$. Let $c_{0}$ be the constant $q_{s}\left(S^{-1} M \beta\right)+f\left(\rho_{S}, \beta\right)+\phi(\beta, \beta)$. Using (II.1.0) we get that

$$
\begin{aligned}
& r(\alpha)=q_{T}\left(T^{-1} N \alpha\right)+f\left(\rho_{T}, N \alpha\right)+\phi\left(\alpha, T^{-1} N \alpha\right)+q_{S}\left(S^{-1} M \alpha\right)+B_{q_{s}}\left(S^{-1} M \alpha, S^{-1} M \beta\right) \\
& \quad+q_{S}\left(S^{-1} M \beta\right)+f\left(\rho_{S}, M \alpha\right)+f\left(\rho_{S}, \beta\right)+\phi\left(\alpha, S^{-1} M \alpha\right)+\phi\left(\beta, S^{-1} M \alpha\right)+\phi(\beta, \beta)+q(\alpha)
\end{aligned}
$$

Now the map $\alpha \rightarrow r(\alpha)+c_{0}$ is a quadratic form by the following considerations. Let $L$ be a linear mapping, $R \in O_{1}(2 m, 2)$. Then $q_{R}$ is a quadratic form and we have that the map $\alpha \rightarrow q_{R}(L(\alpha))$ is a quadratic form as well. This is immediate by (II.1.0).

Furthermore the map $\alpha \rightarrow \phi(\alpha, L(\alpha))$ is also a quadratic form. Indeed, as $L$ is linear and $\phi$ is bilinear we have that

$$
\phi(\alpha, L(\alpha))+\phi(\beta, L(\beta))+\phi(\alpha+\beta, L(\alpha+\beta))=\phi(\beta, L(\alpha))+\phi(\alpha, L(\beta))
$$

hence (II.1.0(ii)) is satisfied; note that (II.1.0(i)) is trivially satisfied.

Finally, functionals over $\mathbf{F}_{2}$ are quadratic forms and if $q$ and $q^{\prime}$ are quadratic forms then the map $\alpha \rightarrow q(\alpha)+q^{\prime}(\alpha)$ is a quadratic form. Now Lemma II.1.6 gives that $\lambda= \pm 2^{e}$ for some integer $e$. This proves the theorem.

Remark. It is possible to determine the element $\beta \in \mathbf{F}_{2}^{2 m}$ of Theorem II.1.7 explicitly. We have that

$$
\begin{array}{r}
\beta=T\left(\rho_{S}\right)+\rho_{T}+\rho_{T S}+T S\left(\left(q_{T}\left(S\left(e_{m+1}\right)\right), q_{T}\left(S\left(e_{m+2}\right)\right), \ldots,\right.\right. \\
\left.\left.q_{T}\left(S\left(e_{2 m}\right)\right), q_{T}\left(S\left(e_{1}\right)\right), \ldots, q_{T}\left(S\left(e_{m}\right)\right)\right)\right) .
\end{array}
$$

This can be derived immediately from the definition of the automorphisms $\mu_{T}$, using that $\mu_{T} \mu_{S}=\mathbf{i}(\beta), \mu_{T S}$ and $q_{T}\left(e_{i}\right)=0$, for all standard basis vectors $e_{i}$ of $\mathbf{F}_{2}^{2 m}$ and all $T \in O_{1}(2 m, 2)$. 
2. Extending D. In this section we investigate under which conditions we can construct an extension of $D$ to $E$. $H$ where $H$ is a subgroup of $\operatorname{Aut}(E)$.

Notation. Let $T, S \in O_{1}(2 m, 2)$. Then $\mu_{T} \mu_{S}=\mathbf{i}(\beta) \mu_{T S}$ for a unique $\beta \in \mathbf{F}_{2}^{2 m}$; for convenience we denote this $\beta$ by $\omega(T, S)$. Hence $\mathbf{i}(\omega()):, O_{1}(2 m, 2) \times O_{1}(2 m, 2) \rightarrow$ $\operatorname{Inn}(E)$ is a well-defined map. We recall that the kernel of a projective representation $R: X \rightarrow G L(n, \mathbb{C})$ of a group $X$ is defined to be the set $\left\{x \in X: R(x)=v . I, v \in \mathbb{C}^{*}\right\}$.

Definition. Let $T \in O_{1}(2 m, 2)$. Define $\Xi\left(\mu_{T}\right)=\sqrt{2}^{-\mathrm{co}_{T}} . P\left(\mu_{T}\right)$.

Note. We have that

$$
\begin{gathered}
\Xi\left(\mu_{T}\right) D(x) \Xi\left(\mu_{T}\right)^{-1}=\Xi\left(\mu_{T}(x)\right) \text { for all } x \in E, \quad T \in O_{1}(2 m, 2) ; \\
\operatorname{det}\left(\Xi\left(\mu_{T}\right)\right)= \pm 1 \text { for all } T \in O_{1}(2 m, 2), \text { and } \Xi\left(\mu_{1}\right)=\Xi(1)=I_{2^{m}}
\end{gathered}
$$

TheOREM II.2.1. Let $G=E$. Aut $(E)$ where $\operatorname{Aut}(E) \subseteq G$ operates in the natural way on $E$. The map $\hat{\Xi}: G \rightarrow G L\left(2^{m}, \mathbb{C}\right)$ defined by $\hat{\Xi}\left(x \mathbf{i}(\gamma) \mu_{T}\right)=D(x) D\left(x^{\gamma}\right) \Xi\left(\mu_{T}\right)$ is a projective representation of $E$. Aut $(E)$ which extends $D$. Let $\mathfrak{R}$ be the factor set corresponding to $\hat{\Xi}$. Then $\Re(x, y)= \pm 1$ for all $x, y \in G$. Moreover $\operatorname{Ker}(\hat{\Xi}) \subseteq E$. Inn $(E)$.

Proof. It is clear by the note preceding Theorem II.2.1 that $\hat{\Xi}$ is a projective representation extending $D$. We now prove the statement concerning $\mathfrak{R}$.

As $\mathfrak{N}\left(x e, y e^{\prime}\right)=\mathfrak{N}(x, y)$ for all $x, y \in G$ and $e, e^{\prime} \in E$ (see [4, Theorem 11.7]) we only need to describe $\mathfrak{N}\left(\mathbf{i}(\gamma) \mu_{T}, \mathbf{i}(\rho) \mu_{S}\right)$ with $\gamma, \rho \in \mathbf{F}_{2}^{2 m}$ and $T, S \in O_{1}(2 m, 2)$. Let $T, S \in$

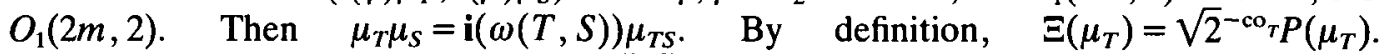
Furthermore $P\left(\mu_{T}\right) P\left(\mu_{S}\right)= \pm 2^{e} D\left(x^{\omega(T, S)}\right) P\left(\mu_{T S}\right) \quad$ and $\operatorname{det}\left(D\left(x^{\omega(T, S)}\right)\right)= \pm 1$, so $\operatorname{det}\left(P\left(\mu_{T}\right)\right) \cdot \operatorname{det}\left(P\left(\mu_{S}\right)\right)= \pm 2^{e .2^{m}} \cdot \operatorname{det}\left(P\left(\mu_{T S}\right)\right)$. Therefore, invoking Theorem II.1.5, we conclude that $e=1 / 2\left(\operatorname{co}_{T}+\operatorname{co}_{S}-\operatorname{co}_{T S}\right)$. Hence $\hat{\Xi}\left(\mu_{T}\right) \hat{\Xi}\left(\mu_{S}\right)= \pm \hat{\Xi}\left(\mu_{T} \mu_{S}\right)$.

Furthermore,

$$
\begin{aligned}
\hat{\Xi}\left(\mathbf{i}(\gamma) \mu_{T}\right) \hat{\Xi}\left(\mathbf{i}(\rho) \mu_{S}\right) & =D\left(x^{\gamma}\right) \cdot \hat{\Xi}\left(\mu_{T}\right) \cdot D\left(x^{\rho}\right) \hat{\Xi}\left(\mu_{S}\right)=D\left(x^{\gamma}\right) \cdot D\left(\mu_{T}\left(x^{\rho}\right)\right) \hat{\Xi}\left(\mu_{T}\right) \hat{\Xi}\left(\mu_{S}\right) \\
& = \pm D\left(x^{\gamma}\right) \cdot D\left(\mu_{T}\left(x^{\rho}\right)\right) \hat{\Xi}\left(\mu_{T} \mu_{S}\right),
\end{aligned}
$$

whereas

$$
\begin{aligned}
\hat{\Xi}\left(\mathbf{i}(\gamma) \mu_{T} \mathbf{i}(\rho) \mu_{S}\right) & =\hat{\Xi}\left(\mathbf{i}(\gamma) \mathbf{i}(T(\rho)) \mu_{T} \mu_{S}\right)=\hat{\Xi}\left(\mathbf{i}(\gamma+T(\rho)) \mu_{T} \mu_{S}\right) \\
& =\hat{\Xi}\left(\mathbf{i}(\gamma+T(\rho)+\omega(T, S)) \mu_{T S}\right)=D\left(x^{\gamma+T(\rho)+\omega(T, S)}\right) \hat{\Xi}\left(\mu_{T S}\right) \\
& = \pm D\left(x^{\gamma+T(\rho)}\right) D\left(x^{\omega(T, S)}\right) \hat{\Xi}\left(\mu_{T S}\right)= \pm D\left(x^{\gamma+T(\rho)}\right) \hat{\Xi}\left(\mu_{T} \mu_{S}\right) \\
& \left.= \pm D\left(x^{\gamma}\right) \cdot D\left(\mu_{T}\left(x^{\rho}\right)\right) \cdot \hat{\Xi}\left(\mu_{T} \mu_{S}\right) \quad \text { (by definition of } \mu_{T}\right) .
\end{aligned}
$$

We see that $\mathfrak{R}\left(\mathbf{i}(\gamma) \mu_{T}, \mathbf{i}(\rho) \mu_{S}\right)= \pm 1$. Now we prove the last statement of the theorem. Suppose $y=x \mathbf{i}(\gamma) \mu_{T}, y \in \operatorname{Ker}(\hat{\Xi})$. Then $\hat{\Xi}\left(x \mathbf{i}(\gamma) \mu_{T}\right)=v . I_{2 m}$ with $v \in \mathbb{C}^{*}$. So we have by definition of the matrices $P\left(\mu_{T}\right)$ that

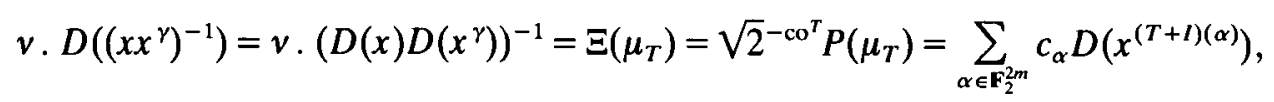

with $c_{\alpha} \in \mathbb{C}^{*}$. By the linear dependence of the matrices $D\left(x^{\alpha}\right)$, with $\alpha \in \mathbf{F}_{2}^{2 m}$, we have that $\operatorname{Im}(T+I)=0$, so $T=I$ and $y \in E . \operatorname{Inn}(E)$. This proves the theorem. 
We now restrict our attention to subgroups of $\operatorname{Aut}(E)$ with odd order. Let $\mathbf{H}$ be a subgroup of $O_{1}(2 m, 2)$ of odd order and suppose $T \in \mathbf{H}$. Then $\prod_{L \in \mathbf{H}} \mathbf{i}(\omega(T, L)) \in \operatorname{Inn}(E)$, so there is a unique $\kappa(T) \in \mathbf{F}_{2}^{2 m}$ such that $\mathbf{i}(\kappa(T))=\prod_{L \in \mathbf{H}} \mathbf{i}(\omega(T, L))$. We now have that $\mathbf{i}(\kappa(T)) \mu_{T} \mathbf{i}(\kappa(S)) \mu_{T}^{-1}=\mathbf{i}(\omega(T, S))^{|\mathbf{H}|} . \mathbf{i}(\kappa(T S))$; this is not difficult to see. As $|\operatorname{Inn}(E)|=$ $2^{2 m}$ and $\mathbf{H}$ has odd order we conclude that there is an odd integer $b$ such that

$$
\mathbf{i}(\omega(T, S))=\left(\mathbf{i}(\kappa(T)) \mu_{T} \mathbf{i}(\kappa(S)) \mu_{T}^{-1} . \mathbf{i}(\kappa(T S))^{-1}\right)^{b}=\mathbf{i}(\kappa(T)) \mu_{T} \mathbf{i}(\kappa(S)) \mu_{T}^{-1} . \mathbf{i}(\kappa(T S))
$$

(note that $\operatorname{Inn}(E)$ is an elementary abelian 2-group). Using this we derive that

$$
\overleftarrow{\mathbf{H}}:=\left\{\mathbf{i}(\kappa(T)), \mu_{T} \mid T \in \mathbf{H}\right\} \text { is a group with } \check{\mathbf{H}} \cap \operatorname{Inn}(E)=\{1\} .
$$

Let $X$ be any subgroup of odd order of $\operatorname{Aut}(E)$, then $X \cap \operatorname{Inn}(E)=\{1\}$ and $X$ is isomorphic to a subgroup $\mathbf{X}$ of $O_{1}(2 m, 2)$. By the Schur-Zassenhaus theorem applied to the group $\operatorname{Inn}(E) \cdot \overrightarrow{\mathbf{X}} \supset X$ we find that $X$ is $\operatorname{Inn}(E)$-conjugate to $\overline{\mathbf{X}}$ in $\operatorname{Inn}(E) \cdot \overline{\mathbf{X}}$.

We proceed with proving a fruitful result on vector spaces over a field of characteristic 2 . Besides that we will give an argument showing that for any $T \in O_{1}(2 m, 2)$ with odd order there exists a $\rho_{T}$ such that $f\left(\rho_{T}, \theta\right)=0$ for all $\theta \in \operatorname{Im}(T+I)$.

LemMA I1.2.3. Let $V$ be a vector space over a field $K$ of characteristic 2. Let $T$ be an operator on $V$. Suppose that $T^{2 k+1}=I$ for some integral $k$. Then $V=\operatorname{Fix}(T) \oplus \operatorname{Im}(T+I)$.

Proof. We know by the dimension theorem on linear mappings that

$$
\operatorname{dim}(\operatorname{Fix}(T))=\operatorname{dim}(\operatorname{Ker}(T+I))=\operatorname{dim}(V)-\operatorname{dim}(\operatorname{Im}(T+I)) .
$$

Furthermore $\operatorname{Im}(T+I) \cap \operatorname{Ker}(T+I)=\{0\}$ as can be seen in the following way. Suppose that $y \in \operatorname{Im}(T+I) \cap \operatorname{Ker}(T+I)$. Then there is some $x$ such that $y=T x+x$ and $(T+I)(T+I)(x)=0$. Now $K$ is of characteristic 2 so $\left(T^{2}+I\right)(x)=0$. So, by $T^{2 k+1}=I$, it follows that $T x=x$ whence $y=0$. This proves the lemma.

COROLlary. Let $V$ be a non-degenerate symplectic vector space over a field of characteristic 2. Let $T$ be a symplectic transformation of odd order. Then $V=$ $\operatorname{Fix}(T) \perp \operatorname{Im}(T+I)$; moreover $\operatorname{Fix}(T)=\operatorname{Im}(T+I)^{\perp}$.

Proof. Let $\langle$,$\rangle denote the symplectic form on V$. If $T v=v$ then $\langle v, T+I(w)\rangle=$ $\langle v, T w\rangle+\langle v, w\rangle=\left\langle T^{-1} v, w\right\rangle+\langle v, w\rangle=0$. So $\operatorname{Fix}(T) \subseteq \operatorname{Im}(T+I)^{\perp}$. As $V$ is nondegenerate we have that $\operatorname{dim}\left(\operatorname{Fix}(T)^{\perp}\right)+\operatorname{dim}(\operatorname{Fix}(T))=\operatorname{dim} V$. By Lemma II.2.3 we conclude that $\operatorname{Fix}(T)=\operatorname{Im}(T+I)^{\perp}$.

We return to one of our earlier results. Consider the proof of Theorem II.1.3. In case of $T \in O_{1}(2 m, 2)$ having odd order we can extend the basis of $F / Z(E)$, given in the proof of Lemma II.1.3, by a basis $\mathscr{B}$ of $\operatorname{Im}(T+I)$ to a basis $\mathscr{B}^{\prime}$ of $E / Z(E)$ and choose $\rho_{T}$ such that

$\mathbf{i}\left(\rho_{T}\right)\left(x^{\alpha}\right)=c \cdot x^{\alpha} ; \quad \mathbf{i}\left(\rho_{T}\right)\left(x^{\beta_{i}}\right)=x_{\beta_{i}}, \quad 1 \leq i \leq n ; \quad \mathbf{i}\left(\rho_{T}\right)\left(x^{\gamma_{i}}\right)=x^{\gamma_{i}} \quad$ if $\quad x^{\gamma_{i}} \cdot Z(E) \in \mathscr{B}$.

If we now look at the expression for $P\left(\mu_{T}\right)$ we see that the exponent of -1 can be described independently of $\rho_{T}$. Indeed, if $(T+I)(\sigma)=0$ then $\sigma \in \operatorname{Fix}(T)$ and the exponent is 0 . If $(T+I)(\sigma) \neq \sigma$ we may assume by the previous lemma and (II.1.4.1) that $\sigma \in \operatorname{Im}(T+I)$. The exponent of -1 is then $q_{T}(\sigma)+f\left(\rho_{T}, T(\sigma)\right)+\phi(T \sigma+\sigma, \sigma)=$ 
$q_{T}(\sigma)+\phi(T \sigma+\sigma, \sigma)$, this follows as $T(\sigma) \in \operatorname{Im}(T+I)$ and $f\left(\rho_{T}, \theta\right)=0$ for all $\theta \in$ $\operatorname{Im}(T+I)$ by choice of $\rho_{T}$. We conclude

Theorem II.2.4. Let $T \in O_{1}(2 m, 2)$ with $\operatorname{order}(T)$ odd. Let $P\left(\mu_{T}\right)$ be defined as in (II.1.3.0) (so $P\left(\mu_{T}\right)^{-1} D(x) P\left(\mu_{T}\right)=D\left(\mu_{T}^{-1}(x)\right)$ holds). Then $\rho_{T}$ can be chosen such that

$$
P\left(\mu_{T}\right)=\sum_{\alpha \in \operatorname{Im}(T+I)}(-1)^{q_{T}(\alpha)+\phi(T \alpha+\alpha, \alpha)} \cdot D\left(x^{(T+I)(\alpha)}\right) .
$$

Let $\mathbf{H}$ be a subgroup of $O_{1}(2 m, 2)$ of odd order. Choose $\left\{\mu_{T} \mid T \in \mathbf{H}\right\}$ such that every $\mu_{T}$ satisfies Theorem II.2.4. Define $\mathbf{H}$ as in II.2.2 and the projective representation of $E . \hat{\mathbf{H}}$ as in Theorem II.2.1. By. Theorem II.2.1 we have that $\mathfrak{R}(x, y)= \pm 1$ for all $x, y \in E$. $\overleftarrow{\mathbf{H}}$. In particular $\mathfrak{R}(x, y)^{|\dot{\mathbf{H}}|}=\mathfrak{N}(x, y)$ for all $x, y \in \overleftarrow{\mathbf{H}}$.

Definition II.2.5. For $x \in \overleftarrow{\mathbf{H}}$ define $\operatorname{sign}(x)=\prod_{z \in \overline{\mathbf{H}}} \mathfrak{N}(x, z)$.

With this definition we have that $\operatorname{sign}(x) \operatorname{sign}(y)=\mathfrak{N}(x, y)^{|\overline{\mathbf{H}}|} \operatorname{sign}(x y)=$ $\mathfrak{N}(x, y) \operatorname{sign}(x y)$. We have therefore linearized the cocycle $\mathfrak{R}$.

For convenience we denote $\operatorname{sign}\left(\mathbf{i}(\kappa(T)) \mu_{T}\right)$ by $\operatorname{sign}(T)$. We are now able to describe representations of $E$. $H$, extending $D$, whenever $H$ has odd order. follows.

This description is one of the major results of this paper and can be formulated as

THEOREM 1I.2.6. Let $E . H$ be a semidirect product of an extraspecial normal 2-subgroup $E$ of first type with a group $H$ of odd order such that $Z(E) \subseteq Z(E . H)$. Suppose $C_{H}(E)=\{1\}$.

Then $H$ is isomorphic with a subgroup of $O_{1}(2 m, 2)$ say $\mathbf{H}$. Choose $\left\{\mu_{r} \mid T \in \mathbf{H}\right\}$ such as to satisfy Theorem II.2.4 and define $\mathbf{H}$ as in (II.2.2). There is some $\mathbf{i}(\gamma) \in \operatorname{Inn}(E)$ such that $E . H \cong E .(\overline{\mathbf{H}})^{\mathbf{i}(\gamma)}$. Define the map $\hat{\Xi}$ on $E .(\overline{\mathbf{H}})$ by

$$
\left.\hat{\Xi}(t)=\operatorname{sign}(T)\left(\frac{1}{2} \sqrt{2}\right)^{\mathrm{co}_{T}} D(y) D\left(x^{\kappa(T)}\right) \sum_{\alpha \in \operatorname{Im}(T+1)}(-1)^{q_{T}(\alpha)+\phi(T \alpha+\alpha, \alpha)} D\left(x^{(T+1)(\alpha)}\right)\right)
$$

where $t=y \mathbf{i}(\kappa(T)) \mu_{T}, y \in E, T \in \mathbf{H}$.

Then $\hat{\Xi}$ is a faithful representation of $E .(\overline{\mathbf{H}})$ extending $D$, and so the map $\Phi$ defined on $E \cdot(\overline{\mathbf{H}})^{i(\gamma)}$ by

$$
\Phi(s)=D\left(x^{\gamma}\right)^{-1} \hat{\Xi}(\mathbf{i}(\gamma) s \mathbf{i}(\gamma)) D\left(x^{\gamma}\right)
$$

is a faithful representation of $E .(\overleftarrow{\mathbf{H}})^{\mathbf{i}(\gamma)}$ (and therefore $\left.E . H\right)$ extending $D$.

Proof. As $D$ is a faithful representation of $E$, we conclude from Theorem II.2.1 that $\hat{\Xi}$ is faithful. The other statements are clear.

3. Extraspecial 2-groups of the second type. In the previous sections we have treated the extension problem for extraspecial groups of the first type i.e. central products of dihedral groups of order 8.

Now we try to find analogous results in case we are dealing with extraspecial 2-groups which are of the second type; i.e. groups which are central product of a group of the first 
type and a quaternion group of order 8 . We will show that essentially all what we have done in the previous sections holds for these groups as well, once we replace $O_{1}(2 m, 2)$ by $\mathrm{O}_{2}(2 m, 2)$; (see Remark II.3.4).

In this section $E$ will always denote an extraspecial 2-group of the second type.

The structure of $E$ can be described as follows. $E$ contains $2 m-2$ involutions $x_{1}, x_{2}, \ldots, x_{m-1}, x_{m+1}, \ldots, x_{2 m-1}$ and elements $x_{m}, x_{2 m}$ of order 4 such that $\langle c\rangle=Z(E)$, $c^{2}=1, x_{i}^{2}=c$ if $i=m$ or $i=2 m$.

The non-trivial commutator relations among the $x_{i}$ are given by $\left[x_{m}, x_{2 m}\right]=$ $\left[x_{i}, x_{m+i}\right]=c$ for $1 \leq i \leq m-1$. Suppose $\alpha \in \mathbf{F}_{2}^{2 m}$ then there exists a unique element $x^{\alpha}=x_{1}^{a_{1}} x_{2}^{a_{2}} \ldots x_{2 m}^{a_{2 m}}$ where $a_{i}=0$ or $1,1 \leq i \leq 2 m$, such that $\alpha=\left(\bar{a}_{1}, \bar{a}_{2}, \ldots, \bar{a}_{2 m}\right)$, with $\bar{a}:=a \bmod 2$. Any $x \in E$ can be written uniquely in the following way

$$
x=c^{a_{0}} x_{1}^{a_{1}} x_{2}^{a_{2}} \ldots x_{2 m}^{a_{2 m}} \text { where } 0 \leq a_{i} \leq 1 \text { for all } i .
$$

Definition II.3.0. We define the bilinear form $\phi^{*}$ on $\mathbf{F}_{2}^{2 m} \times \mathbf{F}_{2}^{2 m}$ by

$$
\phi^{*}(\alpha, \beta)=\left(\sum_{i=1}^{m} a_{m+i} b_{i}\right)+a_{2 m} b_{2 m}+a_{m} b_{m}
$$

Lemma II.3.1. Let $\alpha, \beta \in \mathbf{F}_{2}^{2 m}$. Then $x^{\alpha} \cdot x^{\beta}=c^{\phi^{*}(\alpha, \beta)} x^{\alpha+\beta}$.

Proof. We have $x^{\alpha} \cdot x^{\beta}=c^{\sum m_{1} a_{m+i} b_{i}} \cdot x_{1}^{a_{1}+b_{1}} x_{2}^{a_{2}+b_{2}} \ldots x_{2 m}^{a_{2 m}+b_{2 m}}$.

The $a_{i}$ 's and $b_{i}$ 's, $1 \leq i \leq 2 m$, are 0 or 1 . The $x_{i}$ 's are involutions except if $i=m$ or $2 m$ and therefore $x_{1}^{a_{1}+b_{1}} x_{2}^{a_{2}+b_{2}} \ldots x_{2 m}^{a_{2 m}+b_{2 m}}=x^{\alpha+\beta}$ unless $a_{m}+b_{m}=2$ or $a_{2 m}+b_{2 m}=2$. In the first case $x_{m}^{a_{m}+b_{m}}=c=c^{a_{m} b_{m}}$ and in the second case $x_{2 m}^{a_{2 m}+b_{2 m}}=c=c^{a_{2 m} b_{2 m}}$. The lemma is proved.

The group $E$ can be written as $Q F$ where $Q=\left\langle x_{m}, x_{2 m}\right\rangle, F=\left\langle x_{i}\right| i=1, \ldots, m-$ $1, m+1, \ldots, 2 m-1\rangle$ is a group of first type. In Section 0 we showed how the unique irreducible faithful representation $S$ of $F$ can be described. Now $Q$ has a unique irreducible faithful representation $T$ given by

$$
T\left(x_{m}\right)=i \cdot\left(\begin{array}{ll}
0 & 1 \\
1 & 0
\end{array}\right), \quad T\left(x_{2 m}\right)=i \cdot\left(\begin{array}{rr}
1 & 0 \\
0 & -1
\end{array}\right)
$$

Notation. We define by $A^{*}$ the matrix $\bar{A}^{\prime}$ where $\bar{A}$ is the complex conjugate of the square matrix $A$.

THEOREM II.3.2. Define $D(x y)=T(x) \otimes S(y)$, the Kronecker product of the matrices $T(x)$ and $S(y)$, when $x \in Q$ and $y \in F$. Then $D$ is a well-defined faithful irreducible representation of $E$. Moreover $D^{*}(e)=D\left(e^{-1}\right)$ for all $e \in E$, and $\operatorname{det}(D(e))=1$ for all $e \in E$.

Proof. The map $D$ is well defined, irreducible and faithful as $T$ and $S$ agree on $F \cap Q=Z(E)$ and $F$ and $Q$ centralize each other. Now $(D(x y))^{*}=(T(x) \otimes S(y))^{*}=$ $T^{*}(x) \otimes S^{*}(y)$ [by definition of Kronecker product] $=T\left(x^{-1}\right) \otimes S\left(y^{-1}\right)=D\left(x^{-1} y^{-1}\right)=$ $D\left((x y)^{-1}\right)$ for all $x \in Q, y \in F$. Moreover $\operatorname{det}(D(x y))=\operatorname{det}(T(x))^{\operatorname{deg}(S)} \cdot \operatorname{det}(S(y))^{\operatorname{deg}(T)}$; but $\operatorname{deg}(T)=2, \operatorname{det}(T(x))=1$ for all $x \in Q$, and $\operatorname{det}(S(y))= \pm 1$ as $F / F^{\prime}$ is an elementary abelian 2-group, so $\operatorname{det}(D(x y))=1$ for all $x \in Q, y \in F$. The theorem is proved. 
ReMARK. For extraspecial 2-groups $E$ of the first type we have shown that $D(x) \in G L\left(2^{m}, \mathbb{R}\right)$ for all $x \in E$ (Theorem II.0.1). We lose this property in case $E$ is of the second type. In fact it can be shown (with [4, Cor. 4.15]) that $D$ is not even similar to a real representation. The following results show that the methods in the previous sections need only some slight modifications to hold for the groups that we are studying now.

LemmA II.3.3. Let $\theta$ be an element of Aut $(E)$. Then $\theta\left(x^{\alpha}\right)=t . x^{\alpha^{\prime}}$ for some $t$ in $Z(E)$. The map $T: \alpha \rightarrow \alpha^{\prime}$ is a symplectic transformation preserving the quadratic form $q^{*}$ defined by $q^{*}\left(\alpha_{1}, \alpha_{2}, \ldots, \alpha_{2 m}\right)=\sum_{i=1}^{m} \alpha_{m+i} \alpha_{i}+\left(\alpha_{m}\right)^{2}+\left(\alpha_{2 m}\right)^{2}$.

Proof. Same argument as in Lemma II.1.1 replacing $\phi$ by $\phi^{*}$.

REMARK II.3.4. We see that every element of $\operatorname{Aut}(E)$ induces a linear transformation on the $2 m$-dimensional $\mathbf{F}_{p}$-vectorspace $E / Z(E)$ which preserves the quadratic form $q^{*}$. Denote the group of linear transformations on a $2 m$-dimensional vectorspace which preserve the quadratic form $q^{*}$ by $O_{2}(2 m, 2)$. Note that $\phi^{*}(\alpha, \beta)+\phi^{*}(\beta, \alpha)=f(\alpha, \beta)$ and $\phi^{*}(\alpha, \alpha)=q^{*}(\alpha)$.

Theorem II.3.5. Let $T \in O_{2}(2 m, 2)$. Define the map $\mu_{T}^{\prime}: E \rightarrow E$ by

$$
\mu_{T}^{\prime}\left(c^{a_{0}} x_{1}^{a_{1}} x_{2}^{a_{2}} \ldots x_{2 m}^{a_{2 m}}\right)=c^{a_{0}} c^{q_{r}(\alpha)} x_{1}^{a_{1}} x_{2}^{a_{2}^{\prime}} \ldots x_{2 m}^{a_{2 m}^{\prime}}
$$

where $T(\alpha)=T\left(a_{1}, a_{2}, \ldots, a_{2 m}\right)=\left(a_{1}^{\prime}, a_{2}^{\prime}, \ldots, a_{2 m}^{\prime}\right)$, and $q_{T}(\alpha)=\sum_{i<j} \phi^{*}\left(k_{i}, k_{j}\right) a_{i} a_{j}$, with $k_{i}=T\left(e_{i}\right)$ where $\left\{e_{i}=(\underbrace{0, \ldots, 0}_{i-1}, 1,0, \ldots, 0) \mid 1 \leq i \leq 2 m\right\}$ is the standard basis of $\mathbf{F}_{2}^{2 m}$.

Then $\mu_{T}^{\prime}$ is an element of $\operatorname{Aut}(E)$ and it induces $T$ as a linear transformation on $E / Z(E)$.

Proof. Mimic the proof of Theorem II.1.2. Working out $\phi^{*}(T(\alpha), T(\beta))$ gives

$$
\begin{aligned}
\phi^{*}(T(\alpha), T(\beta))= & \phi^{*}\left(\sum a_{i} T\left(e_{i}\right), \sum b_{i} T\left(e_{i}\right)\right)=\sum_{i, j} \phi^{*}\left(T\left(e_{i}\right), T\left(e_{j}\right)\right) a_{i} b_{j} \\
= & \sum_{i<j}\left(\phi^{*}\left(T\left(e_{i}\right), T\left(e_{j}\right)\right) a_{i} b_{j}+\phi^{*}\left(T\left(e_{j}\right), T\left(e_{i}\right)\right) a_{j} b_{i}\right)+a_{m} b_{m}+a_{2 m} b_{2 m} \\
& {\left[\phi^{*}\left(T\left(e_{i}\right), T\left(e_{i}\right)\right)=\phi^{*}\left(e_{i}, e_{i}\right)=0\right.} \\
& \text { unless } \left.i=m \text { or } i=2 m, \text { as } T \in O_{2}(2 m, 2)\right] \\
= & \sum_{i<j} \phi^{*}\left(T\left(e_{i}\right), T\left(e_{j}\right)\right)\left(a_{i} b_{j}+a_{j} b_{i}\right)+\phi^{*}(\alpha, \beta) \\
& {\left[\text { as } \phi^{*}\left(T\left(e_{i}\right), T\left(e_{j}\right)\right)+\phi^{*}\left(T\left(e_{j}\right), T\left(e_{i}\right)\right)=f\left(T\left(e_{j}\right), T\left(e_{i}\right)\right)\right.} \\
= & \left.f\left(e_{j}, e_{i}\right)=0 \text { unless } j-i=m\right] .
\end{aligned}
$$

The rest of the proof is exactly the same.

Lemma II.3.6. Let $T \in O_{2}(2 m, 2)$ and let $\mu_{T}^{\prime}$ be defined as in Theorem II.3.5. Then there exists some $\rho_{T} \in \mathbf{F}_{2}^{2 m}$ such that

(a) $\mathbf{i}\left(\rho_{T}\right) \mu_{T}^{\prime}$ is an automorphism of $E$ which induces $T$

(b) $\mathbf{i}\left(\rho_{T}\right) \mu_{T}^{\prime}(x)=t x$ for some $x$ with $t \in Z(E)$ only if $t=1$.

Proof. Just follow the lines of the proof of Theorem II.1.3. 
From now on we assume elements $\mathrm{i}\left(\rho_{T}\right)$ chosen such as to satisfy Lemma II.3.6, and denote $\mathbf{i}\left(\rho_{T}\right) \mu_{T}^{\prime}$ by $\mu_{T}$.

Theorem II.3.7. Let $T \in O_{2}(2 m, 2)$. Then the matrix $P\left(\mu_{T}\right)$ defined by

$$
P\left(\mu_{T}\right)=\frac{1}{c_{T}} \sum_{\alpha \in \mathbf{F}_{2}^{2 m}}(-1)^{q_{T}(\alpha)+f\left(\rho_{T}, T(\alpha)\right)+\phi^{*}\left(T_{\alpha+\alpha, \alpha)}\right.} \cdot D\left(x^{(r+l)(\alpha)}\right)
$$

is invertible and satisfies $P\left(\mu_{T}\right)^{-1} D(x) P\left(\mu_{T}\right)=D\left(\mu_{T}^{-1}(x)\right)$.

Proof. Apply (TH).

Lemma II.3.8. Let $T \in O_{2}(2 m, 2)$. Then

$$
P\left(\mu_{T}\right)^{*}=A^{-1} P\left(\mu_{T^{-1}}\right) A \text { with } A=D\left(x^{\sigma}\right) \text { for some suitable } \sigma \in \mathbf{F}_{2}^{2 m} .
$$

Proof. Mimic the proof of Lemma II.1.4 using Theorem II.3.2 instead of Corollary II.0.2, and Theorem II.3.6 instead of Theorem II.1.3. Moreover by (II.3.5.1) we see that (II.1.3.1) holds for $T \in O_{2}(2 m, 2)$. This proves the lemma.

TheOREM II.3.9. Let $T \in O_{2}(2 m, 2)$. Then there exists an integer $k$ and a suitable $\gamma \in \mathbf{F}_{2}^{2 m}$, such that $\operatorname{det}\left(P\left(\mu_{T}\right)\right)=i^{k} \cdot 2^{2^{m-1} \cdot \mathrm{co}_{T}}$ and $P\left(\mu_{T}\right) P\left(\mu_{T^{-1}}\right)= \pm 2^{\mathrm{co}_{T}} . D\left(x^{\gamma}\right)$.

Proof. Following the proof of Theorem II.1.5 we obtain that $P\left(\mu_{T}\right) P\left(\mu_{T^{-1}}\right)=$ $\lambda . D\left(x^{\gamma}\right)$ where $\lambda=2^{\mathrm{co} T}$. Now Lemma II.3.8 gives $\left|\operatorname{det}\left(P\left(\mu_{T}\right)\right)\right|^{2}=2^{\mathrm{co} r} \cdot 2^{m}$.

We know already from the construction of $D$ that $D(x) \in G L\left(2^{m}, \mathbb{Z}(i)\right)$ and so, by definition of $P, P\left(\mu_{T}\right) \in G L\left(2^{m}, \mathbb{Z}(i)\right)$. Hence $\operatorname{det}\left(P\left(\mu_{T}\right)\right) \in \mathbb{Z}(i)$. The equation $|y|^{2}=2^{2 r}$ has only the solutions $y \in\left\{2^{r},-2^{r}, i .2^{r},-i .2^{r}\right\}$ in $\mathbb{Z}(i)$. So the theorem is proved.

Although it is possible that $\operatorname{det}\left(P\left(\mu_{T}\right)\right)$ is not a rational integer the cocycle determined by $P$ can be described in the same way as in the case of extraspecial 2-groups of the first type. This could be expected as $P\left(\mu_{T}\right)$ is defined as a linear combination of the matrices $D(x)$ where the scalars in this combination are (as earlier) 1 or -1 , and as these scalars determine completely the values of the cocycle belonging to $P$. Hence this cocycle will have essentially the same range of values as the cocycle that we've investigated earlier, as we can apply the same arguments as in the proof of Theorem II.1.7. So we have the following result.

THEOREM II.3.10. Let $T, S \in O_{2}(2 m, 2)$. Then $\mu_{T} \mu_{S}=h . \mu_{T S}$ where $h$ is an inner automorphism of $E$. Let $h(x)=x^{\beta} x\left(x^{\beta}\right)^{-1}$. Then $P\left(\mu_{T}\right) P\left(\mu_{S}\right)= \pm 2^{e} . D\left(x^{\beta}\right) P\left(\mu_{T S}\right)$ for some integer $e$.

Proof. See the proof of Theorem II.1.7.

We now introduce a projective extension of $D$ in the same way as in Section 2.

THeOREM II.3.11. Let $T \in O_{2}(2 m, 2)$. Define $\Xi\left(\mu_{T}\right)=\sqrt{2}^{- \text {co }}$. $P\left(\mu_{T}\right)$. Then the map $\hat{\Xi}$ defined on $G=E$. Aut $(E)$ by

$$
\hat{\Xi}\left(x \mathbf{i}(\gamma) \mu_{T}\right)=D(x) D\left(x^{\gamma}\right) \Xi\left(\mu_{T}\right)
$$

is a projective representation of $E . \operatorname{Aut}(E)$ extending $D$.

Let $\mathfrak{N}$ be the factor set corresponding to $\hat{\Xi}$. Then $\Re(x, y)= \pm 1$ for all $x, y \in G$. 
Proof. We follow the proof of Theorem II.2.1. We only need to establish that $\hat{\Xi}\left(\mu_{T}\right) \hat{\Xi}\left(\mu_{s}\right)= \pm \hat{\Xi}\left(\mu_{T} \mu_{S}\right)$. This can be done as follows. By Theorem II.3.10P( $\left.\mu_{T}\right) P\left(\mu_{s}\right)=$ $\pm 2^{e} . D\left(x^{\beta}\right) P\left(\mu_{T S}\right)$.

Taking determinants gives $\operatorname{det}\left(P\left(\mu_{T}\right)\right) \operatorname{det}\left(P\left(\mu_{S}\right)\right)=2^{2^{m} \cdot e} \cdot \operatorname{det}\left(P\left(\mu_{T S}\right)\right.$ ) (note that $\operatorname{det}\left(D\left(x^{\beta}\right)\right)=1$ by Theorem II.3.2), and an application of Theorem II.3.9 yields that there are integers $a, b, c$ such that

$$
\left(i^{a} \cdot 2^{2^{m-1} \cdot \operatorname{co}_{T}}\right)\left(i^{b} \cdot 2^{2^{m-1} \cdot \cos _{s}}\right)=2^{2^{m} \cdot e} \cdot\left(i^{c} \cdot 2^{2^{m-1} \cdot \operatorname{co}_{T s}}\right) .
$$

Conclusion: $a+b \equiv c \bmod 4), e=1 / 2\left(\operatorname{co}_{T}+\operatorname{co}_{S}-\operatorname{co}_{T S}\right)$. Hence using the definition of $\hat{\Xi}$, we conclude that $\hat{\Xi}\left(\mu_{T}\right) \hat{\Xi}\left(\mu_{S}\right)= \pm \hat{\Xi}\left(\mu_{T} \mu_{S}\right)$. The proof of Theorem II.3.11 is complete.

We construct $\overline{\mathbf{H}}$, where $\mathbf{H}$ is a subgroup of odd order of $O_{2}(2 m, 2)$, in the same way as in (II.2.2). Furthermore we define

$$
\phi_{E}=\left\{\begin{array}{l}
\phi \text { if } E \text { is extraspecial of the first type } \\
\phi^{*} \text { if } E \text { is extraspecial of the second type. }
\end{array}\right.
$$

Now it is clear that the analogue of Theorem II.2.4 holds with $O_{2}(2 m, 2)$ instead of $O_{1}(2 m, 2)$, so we arrive at the following theorem.

Theorem II.3.12. Let $T \in O_{2}(2 m, 2)$ with order $(T)$ odd. Let $P\left(\mu_{T}\right)$ be defined as in (II.1.3.0). Then $\rho_{T}$ can be chosen such that

$$
P\left(\mu_{T}\right)=\sum_{\alpha \in \operatorname{Im}(T+l)}(-1)^{q_{T}(\alpha)+\phi^{*}(T \alpha+\alpha, \alpha)} \cdot D(x(T+I)(\alpha)) .
$$

The next result is proved along the same lines as in the proof of Theorem II.2.6.

THEOREM II.3.13. Let E. $H$ be a semidirect product of an extraspecial normal 2-subgroup $E$ with a group $H$ of odd order such that $Z(E) \subseteq Z(E . H)$. Suppose $C_{H}(E)=\{1\}$.

Let $E$ be an extraspecial 2-group of $i$ th type $(i=1,2)$. Then $H$ is isomorphic with $a$ subgroup of $O_{i}(2 m, 2)$, say $\mathbf{H}$. Choose $\left\{\mu_{T} \mid T \in \mathbf{H}\right\}$ such as to satisfy Theorem II.2.4 or Theorem II.3.12 as the case may be. There is some $\mathrm{i}(\gamma) \in \operatorname{Inn}(E)$ such that $E . H \cong$ $E .(\overline{\mathbf{H}})^{\mathbf{i}(\gamma)}$. Define the map $\hat{\Xi}$ on $E .(\overline{\mathbf{H}})$ by

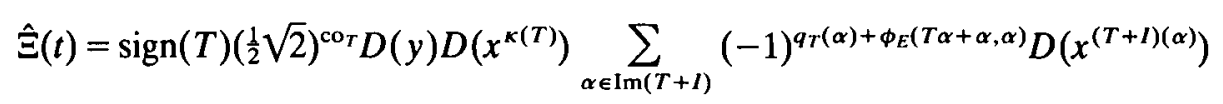

where $t=y \mathbf{i}(\kappa(T)) \mu_{T}, y \in E, T \in \mathbf{H}$.

Then $\hat{\Xi}$ is a faithful representation of $E .(\overleftarrow{\mathbf{H}})$ extending $D$, and so the map $\Phi$ defined on $E .(\overline{\mathbf{H}})^{\mathbf{i}(\gamma)}$ by

$$
\Phi(s)=D\left(x^{\gamma}\right)^{-1} \hat{\Xi}(\mathbf{i}(\gamma) \operatorname{si}(\gamma)) D\left(x^{\gamma}\right)
$$

is a faithful representation of $E .(\overline{\mathbf{H}})^{\mathrm{i}(\gamma)}$ (and therefore of $\left.E . H\right)$ extending $D$.

4. Extending $D$ if the complement has a cyclic Sylow 2-subgroup. Let the Sylow 2-subgroup of $H$ be cyclic. We conclude from [3, Satz IV.2.8] that $H=P K$ where $P$ is a cyclic Sylow 2-subgroup of $H$, and $K$ is a normal subgroup of $H$ satisfying $H / K \cong P$. 
In this section we describe an extension of the unique faithful irreducible character $D_{E}$ of an extraspecial 2-subgroup $E$ to $G=E . H$, under the extra hypothesis that $[E, K]=E$. Note that the structure of $D$ depends strongly on the type of $E$ (see Theorem II.0.1 and Theorem II.3.2). We describe first how $D$ extends to $E$. $C$ if $C$ is a cyclic subgroup of $\operatorname{Aut}(E)$ and show that this extension is faithful if $C \cap \operatorname{Inn}(E)=\{1\}$. We have the following theorem; for the definition of $\hat{\Xi}$ and $\mathfrak{R}($,$) see Theorem II.2.1.$

Theorem II.4.1. Let $C$ be a cyclic 2-subgroup of $\operatorname{Aut}(E)$, of order $2^{n}$. Let $C=\mathbf{i}(\gamma) \mu_{T}$ be a generator of $C$, and let $\mathrm{i}(\zeta) \mu_{s}$ be the unique involution of $C$.

Define $\hat{D}\left(e \cdot c^{m}\right)=D(e) . A^{m}$ for $e \in E$ and $0 \leq m \leq 2^{n}-1$, where

(i) $A=\tau . D\left(x^{\gamma}\right) \hat{\Xi}\left(\mu_{T}\right)$;

(ii) $\tau^{2^{n}}=(-1)^{\phi_{E}(\zeta, \zeta)}$.

Then $\hat{D}$ is a representation of $E$. C extending $D$; if $C \cap \operatorname{Inn}(E)=\{1\}$ then $\hat{D}$ is faithful.

Moreover $\hat{D}\left(c^{m}\right)=\lambda\left(c^{m}\right) . \hat{\Xi}\left(c^{m}\right)$, with $\lambda\left(c^{m}\right)=\tau^{m} . \mathfrak{N}(c, c) \mathfrak{N}\left(c^{2}, c\right) \ldots \mathfrak{N}\left(c^{m-1}, c\right)$.

Proof. Let $\hat{\Xi}$ be the projective representation as defined in Theorem II.2.1. It is evident that the map $R: E . C \rightarrow G L\left(2^{m}, \mathbb{C}\right)$ defined by $R\left(e \cdot c^{m}\right)=D(e) R(c)^{m}$, for $e \in E$, is a representation of $E$. $C$ extending $D$, if and only if there exists $\tau \in \mathbb{C}^{*}$, such that $R(c)=\tau . \hat{\Xi}(c)$ and $(R(c))^{2^{n}}=I$. Now $\hat{\Xi}(c)^{2^{n}}=\varepsilon . I$, so $\tau^{2^{n}}=\varepsilon^{-1}$ in the former situation, and we can determine $\tau$ once we know $\varepsilon$. By Theorem II.2.1 we have that $\left(\hat{\Xi}\left(c^{i}\right)\right)^{2}=$ $\pm \hat{\Xi}\left(c^{2 i}\right)$ for all $i \in \mathbb{N}$; whence $\left(\hat{\Xi}\left(c^{2^{n-1}}\right)\right)^{2}=\varepsilon$. I with $\varepsilon= \pm 1$. As $c^{2^{n-1}}$ is an involution, we must have that $c^{2^{n-1}}=\mathrm{i}(\zeta) \mu_{S}, S=T^{2^{n-1}}, S^{2}=I$. So $S=I$ or $S$ has order 2 .

If $S=I$ then $\hat{\Xi}\left(c^{2^{n-1}}\right)=D\left(x^{\zeta}\right)$, and $\varepsilon=(-1)^{\phi_{E}(\zeta, \xi)}$. So suppose $S$ has order 2 . Then $\left(\hat{\Xi}\left(c^{2^{n-1}}\right)\right)^{2}=D\left(x^{5}\right) D\left(\mu_{S}\left(x^{5}\right)\right)$. $\left(\hat{\Xi}\left(\mu_{S}\right)\right)^{2}$, so

$$
\left(\hat{\Xi}\left(\mu_{S}\right)\right)^{2}=(-1)^{d_{s}} D\left(x^{\zeta+s \zeta}\right) \text { where }\left(\mu_{S}\left(x^{s \zeta}\right)\right)\left(x^{s \zeta}\right)^{-1}=(-1)^{d_{s}} \cdot x^{\zeta+s \zeta} \quad \text { (by II.1.5.1). }
$$

We conclude that

$$
\begin{aligned}
\varepsilon . I & =D\left(x^{\zeta}\right) D\left(\mu_{S}\left(x^{\zeta}\right)\right) D\left(\mu_{S}\left(x^{s \zeta}\right)\right) D\left(x^{s \zeta}\right)^{-1}=D\left(x^{\zeta}\right) D\left(\mu_{S}\left(x^{\zeta} x^{s \zeta}\right)\right) D\left(x^{\zeta} x^{s \zeta}\right)^{-1} D\left(x^{\zeta}\right) \\
& =D\left(x^{\zeta}\right)^{2}, \text { as } S^{2}=I, \quad S \zeta+\zeta \in \operatorname{Fix}(S) .
\end{aligned}
$$

Now $D\left(x^{5}\right)^{2}=(-1)^{\phi_{E}(\zeta, \zeta)}$. I. Hence $\varepsilon=(-1)^{\phi_{E}(\zeta, \zeta)}$. This proves that $\hat{D}$ is a representation of $E . C$ extending $D$. Moreover $\operatorname{Ker}(\hat{D}) \subseteq E . \operatorname{Inn}(E) \cap E . C$ (see Theorem II.2.1); so $C \cap \operatorname{Inn}(E)=\{1\}$ gives that $E . \operatorname{Inn}(E) \cap E . C=E$, whence $\operatorname{Ker}(\hat{D})=\{1\}$ as $D$ is faithful. The last statement is obvious. The theorem is proved.

We will now construct an extension of $D$ to $E$. $H$ with $H \subseteq \operatorname{Aut}(E)$, if $H$ has a cyclic Sylow 2-subgroup $U$, such that the 2-complement $K$ of $H$ (see [3, Satz IV.2.8]), has the property that $[E, K]=E$.

In fact we can prove somewhat more. Let $H$ be 2-nilpotent with Sylow 2-subgroup $U$ and 2-complement $K$. Let $\hat{\Xi}$ be the projective representation of $G=E$. $H$, which extends $D$ (see Theorem II.2.1). We will show how an extension of $D$ to $G$ can be obtained if $\hat{\Xi}$ can be linearized on both $E . U$ and $E . K$, and $[E, K]=E$.

Proposition II.4.2. Let $\mathfrak{N}$ be the cocycle associated to $\hat{\Xi}$. Suppose that functions $\sigma: E . K \rightarrow \mathbb{C}^{*}$ and $\pi: E . U \rightarrow \mathbb{C}^{*}$ exist such that $\mathfrak{N}(x, y)=\sigma(x) \sigma(y)(\sigma(x y))^{-1}$, for all $x, y \in E . K$ and $\mathfrak{R}(x, y)=\pi(x) \pi(y)(\pi(x y))^{-1}$ for all $x, y \in E$. U. Define the map $\hat{\Xi}^{*}$ on $G=E$. $H$ by $\hat{\Xi}^{*}(a b)=(\pi(a))^{-1} \hat{\Xi}(a)(\sigma(b))^{-1} \hat{\Xi}(b)$ for all $a \in U, b \in E$.K. Then $\hat{\Xi}^{*}$ is an irreducible representation of $G$ extending $D$. 
Proof. Theorem II.3.11 shows that the cocycle $\mathfrak{N}$ has values 1 or -1 . We now introduce the set $G^{*}=\{(g, \varepsilon) \mid g \in G, \varepsilon= \pm 1\}$ which is a group under the law of composition defined by $\left(g_{1}, \varepsilon\right)\left(g_{2}, \varepsilon^{\prime}\right)=\left(g_{1} g_{2}, \varepsilon \varepsilon^{\prime} \mathfrak{R}\left(g_{1}, g_{2}\right)\right)$. We have that $E^{*}=$ $\left\{(e, \varepsilon \mid e \in E, \varepsilon= \pm 1\}\right.$ is a normal subgroup of $G^{*}$ and $C=\{(1, \varepsilon) \mid \varepsilon= \pm 1\}$ is a central subgroup of $G^{*}$. Define the map $D^{*}$ on $E^{*}$ by $D^{*}((e, \varepsilon))=D(e)$ for all $e \in E$. Then $D^{*}$ is an irreducible representation of $E^{*}$.

The map $\hat{\Xi}_{0}$ defined by $\hat{\Xi}_{0}(g, \varepsilon)=\varepsilon . \hat{\Xi}(g)$ is an irreducible representation of $G^{*}$ and $\hat{\Xi}_{0 \mid E^{*}}=\theta^{*} . D^{*}$ where $\theta^{*}$ is the invariant linear character of $E^{*}$ defined by $\theta^{*}(e, \varepsilon)=\varepsilon$. Note that $\operatorname{Ker}\left(\theta^{*}\right)=N=\{(e, 1) \mid e \in E\}$. Let $\pi$ and $\sigma$ be as assumed by the proposition. By the assumptions on $\pi$ and $\sigma$, we conclude from the definition of $G^{*}$ that $L:=\left\{\left(r,(\sigma(r))^{-1}\right) \mid r \in E . K\right\}$ is a subgroup of $G^{*}$ isomorphic to $E . K$, and $T:=$ $\left\{\left(t,(\pi(t))^{-1}\right): t \in E . U\right\}$ is a subgroup of $G^{*}$ isomorphic to $E . U$, observe that $N=L \cap$ $T$, as $\pi(x)=\sigma(x)=1$ for all $x \in E$. Furthermore $L \cap C=\{1\}=T \cap C$, so $\theta^{*}$ extends to both $L C$ and $T C$ as $C$ is central.

Now $T C$ is a Sylow 2-group of $G^{*}$, and as $L / N \cong K, L$ contains a Sylow $r$-subgroup of $G^{*}$ for any odd prime $r$. Hence for any prime $p$ there is a group $H_{p}$ such that $\theta^{*}$ extends to $H_{p}$ and $H_{p} / E^{*} \in \operatorname{Syl}_{p}\left(G^{*} / E^{*}\right)$. Hence $\theta^{*}$ extends to $G^{*}$ [4, Theorem 6.26] and $G^{* \prime} \cap C=\{1\}$.

So $C G^{* \prime} / G^{* \prime}$ is cyclic of order 2. As $[E, K]=E$, we have that $N \subseteq[L, N] \subseteq G^{* \prime}$. Hence $L G^{* \prime} / G^{* \prime}=O_{2^{\prime}}\left(G^{*} / G^{* \prime}\right)$, and $C G^{* \prime} \cap L G^{* \prime}=G^{* \prime}$. So $C \notin L G^{* \prime}$ and $L C \cap$ $L G^{* \prime}=L$, as $L$ has index 2 in $L C$. Now $L C$ is normal in $G^{*}$, by construction of $G^{*}$; hence $L$, being an intersection of normal subgroups, is normal in $G^{*}$.

As $L \cap T=N$, we have that $L T$ has index 2 in $G^{*}$. So $C$ is not contained in $L T$, as $T C$ is a Sylow 2-subgroup of $G^{*}$. Hence $C \times L T=G^{*}, L T \cong G$.

Put $X=L T$ say $X=\{(g, \rho(g)) \mid g \in G\}$. As $T \subseteq X$ we see that $\rho(t)=(\pi(t))^{-1}$ for all $t \in E . U$ indeed, if $(t, \rho(t))=\left(t,-(\pi(t))^{-1}\right)$ this would imply that $(1,-1) \in X$, a contradiction. In the same way we get that $\rho(r)=\sigma(r)$ for all $r \in E$. K. As $X=T L$ we conclude that $(t r, \rho(t r))=\left(t,(\pi(t))^{-1}\right)\left(r,(\sigma(r))^{-1}\right)$ if $t \in E . U, r \in E$. K. Hence

$$
\rho(t r)=\mathfrak{R}(t, r)(\pi(t))^{-1}(\sigma(r))^{-1} \quad \text { for all } t \in E . U, \quad r \in E . K .
$$

We have that $\mathfrak{R}(g, h)=(\rho(g))^{-1}(\rho(h))^{-1} \rho(g h)$ as $X$ is a subgroup of $G^{*}$. So

$$
\begin{aligned}
\Re\left(t r, t^{\prime} r^{\prime}\right) & =(\rho(t r))^{-1}\left(\rho\left(t^{\prime} r^{\prime}\right)\right)^{-1} \rho\left(t r t^{\prime} r^{\prime}\right) \\
& =(\mathfrak{N}(t, r))^{-1} \pi(t) \sigma(r)\left(\mathfrak{N}\left(t^{\prime}, r^{\prime}\right)\right)^{-1} \pi\left(t^{\prime}\right) \sigma\left(r^{\prime}\right) \rho\left(t t^{\prime} r^{\prime} r^{\prime}\right) \\
& =\pi(t) \pi\left(t^{\prime}\right)\left(\pi\left(t t^{\prime}\right)\right)^{-1} \sigma(r) \sigma\left(r^{\prime}\right)\left(\sigma\left(r^{t^{\prime}} r^{\prime}\right)\right)^{-1}\left(\mathfrak{N}\left(t^{\prime}, r^{\prime}\right)\right)^{-1}(\mathfrak{N}(t, r))^{-1} \mathfrak{N}\left(t t^{\prime}, r^{t^{\prime}} r^{\prime}\right)
\end{aligned}
$$

We finish the proof of the proposition by showing in a straightforward way that the map $\hat{\Xi}^{*}$ is a well-defined homomorphism. Let $a, a^{\prime} \in U$ and $b, b^{\prime} \in E$. K. Then

$$
\begin{aligned}
\hat{\Xi}^{*}(a b) \hat{\Xi}^{*}\left(a^{\prime} b^{\prime}\right)= & (\pi(a))^{-1} \hat{\Xi}(a)(\sigma(b))^{-1} \hat{\Xi}(b)\left(\pi\left(a^{\prime}\right)\right)^{-1} \hat{\Xi}\left(a^{\prime}\right)\left(\sigma\left(b^{\prime}\right)\right)^{-1} \hat{\Xi}\left(b^{\prime}\right) \\
= & (\pi(a))^{-1}\left(\pi\left(a^{\prime}\right)\right)^{-1}(\sigma(b))^{-1}\left(\sigma\left(b^{\prime}\right)\right)^{-1} \mathfrak{R}(a, b) \mathfrak{N}\left(a^{\prime}, b^{\prime}\right) \hat{\Xi}(a b) \hat{\Xi}\left(a^{\prime} b^{\prime}\right) \\
= & (\pi(a))^{-1}\left(\pi\left(a^{\prime}\right)\right)^{-1}(\sigma(b))^{-1}\left(\sigma\left(b^{\prime}\right)\right)^{-1} \mathfrak{N}(a, b) \mathfrak{R}\left(a^{\prime}, b^{\prime}\right) \\
& \times \mathfrak{N}\left(a b, a^{\prime} b^{\prime}\right) \hat{\Xi}\left(a b a^{\prime} b^{\prime}\right)
\end{aligned}
$$




$$
\begin{aligned}
& \left.=\left(\pi\left(a a^{\prime}\right)\right)^{-1}\left(\sigma\left(b^{a^{\prime}} b^{\prime}\right)\right)^{-1} \mathfrak{R}\left(a a^{\prime}, b^{a} b^{\prime}\right) \hat{\Xi}\left(a b a^{\prime} b^{\prime}\right) \quad \text { (using }(\ddagger)\right) \\
& =\left(\pi\left(a a^{\prime}\right)\right)^{-1} \sigma\left(b^{a^{\prime}} b^{\prime}\right) \hat{\Xi}\left(a a^{\prime}\right) \hat{\Xi}\left(b^{a^{\prime}} b^{\prime}\right) \\
& =\hat{\Xi}^{*}\left(a a^{\prime} b^{a^{\prime}} b^{\prime}\right) \\
& =\hat{\Xi}^{*}\left(a b a^{\prime} b^{\prime}\right) .
\end{aligned}
$$

The proposition is proved.

Let $H$ have a cyclic Sylow 2-subgroup $C$ with 2-complement $K$ such that $[K, E]=E$. Let $\mathbf{H}$ be a subgroup of $O_{i}(2 m, 2)(i=1,2)$ isomorphic to $K$. Let $\mathbf{i}(\gamma)$ be an inner automorphism such that $K \cong(\mathbf{H})^{\mathbf{i}(\gamma)}$ (see II.2.2). Let $\lambda$ and sign be the functions defined in Theorem II.4.1 and Definition II.2.5 respectively. Then $\lambda$ and sign can be extended to functions on $E . C$ viz $E . K$ such that $\left.\lambda\right|_{E}=\left.\operatorname{sign}\right|_{E}=1$. We have that

$\mathfrak{N}\left(b, b^{\prime}\right)=\operatorname{sign}(\mathbf{i}(\gamma) b \mathbf{i}(\gamma))^{-1} \cdot \operatorname{sign}\left(\mathbf{i}(\gamma) b^{\prime} \mathbf{i}(\gamma)\right)^{-1} \cdot \operatorname{sign}\left(\mathbf{i}(\gamma) b b^{\prime} \mathbf{i}(\gamma)\right) \quad$ for all $b, b^{\prime} \in E . K$;

$\mathfrak{N}\left(a, a^{\prime}\right)=(\lambda(a))^{-1}\left(\lambda\left(a^{\prime}\right)\right)^{-1}\left(a a^{\prime}\right)$ for all $a, a^{\prime} \in E . C$.

Hence we finally arrive at the main theorem of this section.

TheOREM II.4.3. Let $H$ have a cyclic Sylow 2-subgroup $C$ with 2-complement $K$ such that $[K, E]=E$. Define the map $\hat{\Xi}^{*}$ on $G=E . H$ by $\hat{\Xi}^{*}(a b)=$ $\lambda(a) \hat{\Xi}(a) \operatorname{sign}(\mathbf{i}(\gamma) b \mathbf{i}(\gamma)) \hat{\Xi}(b)$ for all $a \in C, b \in E . K$. Then $\hat{\Xi}^{*}$ is an irreducible representation of $G$ extending $D$.

\section{REFERENCES}

1. E. C. Dade, Characters of groups with normal extra special subgroups, Math. Z. 152 (1976), $1-31$.

2. R. B. Howlett, Extending characters from normal subgroups, Lecture Notes in Mathematics No. 697 (Springer-Verlag, 1978).

3. B. Huppert, Endliche Gruppen I (Springer-Verlag, 1967).

4. I. M. Isaacs, Character theory of finite groups (Academic Press, 1976).

5. E. B. Kuisch, On the construction of matrix representations and their extensions, Indagationes Mathematicae New Series 1 (1990), to appear.

6. W. Scharlau, Quadratic and hermitian forms (Springer-Verlag, 1985).

FaCULTEIT WisKunde \& INFORMATICA

UNIVERSITEIT VAN AMSTERDAM

Plantage Muidergracht 24

1018 TV AMSTERDAM

The NeTHERLANDS. 